\title{
Direct numerical simulation of homogeneous turbulence in combination with premixed combustion at low Mach number modelled by the $G$-equation
}

\author{
By T. C. TREURNIET, F. T. M. NIEUWSTADT $\dagger$ \\ AND B. J. BOERSMA† \\ J. M. Burgers Centre, Delft University of Technology, Leeghwaterstraat 21, 2628 CA, Delft, \\ The Netherlands
}

(Received 27 January 2002 and in revised form 24 February 2005)

We consider the direct numerical simulation (DNS) of a homogeneously turbulent flow in combination with a premixed flame. The combustion takes place in the flamelet regime which means that combustion occurs in a very thin layer, called the flame front. The position of the flame front is modelled by means of the $G$-equation, in which the flame front is represented by an isosurface $G_{0}$ of a scalar field $G(\boldsymbol{x}, t)$. The flow is described by the Navier-Stokes equations in the low-Mach-number limit, which allows for the inclusion of gas expansion due to the temperature increase by the combustion. The advantage of the low-Mach-number approximation is that efficient numerical methods, used for incompressible flows, can be applied to solve the discretized equations.

The calculations are carried out in a box with homogeneous isotropic turbulence. In addition, a uniform mean velocity is imposed with a inflow boundary condition at $x=0$. The inflow velocity is adjusted such that the mean position of the flame is stabilized at a fixed position. This allows us to use time averaging to obtain accurate statistics, which are very difficult to obtain when the flame is allowed to propagate. In the $y$-and $z$-direction, periodic boundary conditions are applied.

The numerical code has been checked with a well-known theoretical result, the so-called Darrieus-Landau instability of a thin flame front. The results show a good agreement between the computed growth rate and the theoretical value when the thickness of the flame front is much smaller than the wavelength of the disturbance. When this condition is not met, the growth rate becomes lower than the theory in agreement with the restriction under which the theory is valid.

For the computations in homogeneous turbulence, the results show an increase in the turbulent flame speed with increasing turbulent intensity at the position of the flame front. This is in good agreement with experimental data and theory. The turbulent flame speed shows also an increase as a function of the heat release parameter. This is because disturbances on the flame front, induced by the turbulence, are enhanced by the Darrieus-Landau instability.

The budgets of the turbulent kinetic energy and the enstrophy show that the expansion of the gas across the flame front suppresses the turbulence. At higher expansion rates, turbulence in the direction of the mean velocity increases and as a result turbulence becomes strongly anisotropic. The increase is due to two processes. 
The first is the influence of the Darrieus-Landau instability already mentioned. The second is the baroclinic production of vorticity owing to the flame front density and pressure gradients not being aligned.

\section{Introduction}

Premixed combustion occurs when fuel and oxidizer are already fully mixed before ignition. When such a mixture is ignited, the combustion takes place in a flame front which propagates through the mixture. When the chemical time scale is very short with respect to the diffusion time, this flame front will be very thin.

In this study, we shall limit ourselves to combustion by deflagration for which the mechanism of flame-front propagation is as follows. The thermal energy, which is released during combustion, will heat up the unburnt gas in front of the flame by means of diffusion. As soon as the ignition temperature is reached, this unburnt gas will start to burn. So the flame propagates by diffusion through the unburnt mixture. The speed of such a deflagration flame in a laminar flow is rather modest, i.e. of the order of several metres per second.

In the case of deflagration in a turbulent flow, the situation is more complicated. Since the fuel and oxidizer are already fully mixed, turbulence is not relevant in this case for mixing, but turbulence will interact with the flame front during its propagation through the medium. This has three effects on the flame propagation.

The first effect (Damköhler 1940) is that turbulent fluctuations will distort the flame front, giving a larger flame surface. The result is an increased combustion rate and an increase of the mean flame speed. This effect has been confirmed by experiments. For instance, it is known that in the case of gas explosions, the combustion rate increases strongly in the turbulent wake behind obstacles (e.g. Ibrahim, Hargrave \& Williams 2001).

The second effect is related to flame-front instabilities. Williams (1985b) gives an overview of all instabilities that can occur in combustion. For our application, only hydrodynamic instabilities and diffusive instabilities are relevant. An example of the former is the Darrieus-Landau instability. Landau (1944) showed that thin planar deflagration flames are unconditionally unstable, as a result of the density gradient in combination with the acceleration of the flow. Diffusive instabilities occur when the reactants have different diffusivities. This type of instability is observed mainly in oxygen-hydrogen flames. Turbulence in the flow field can trigger these instabilities, i.e. small disturbances in the flame front, induced by the turbulence may grow further owing to these instabilities. This causes the area of the flame front to increase strongly. We refer to Markstion (1964), Williams (1985b) and Zoldovich et al. (1985) for further discussion on instabilities.

The third effect of flame-front/turbulence interaction is the production of vorticity at the position of the flame front. This is due to the non-alignment of the pressure and the density gradient and is called baroclinic vorticity production. This non-alignment occurs when the flame front is distorted from its planar shape by either turbulence or instability processes. This effect has been studied by Rutland \& Ferziger (1991), who carried out a two-dimensional simulation. Mueller et al. (1998) performed particle image velocimetry (PIV) measurements of the interaction between a vortex pair and a premixed flame, which provided the first quantitative experimental results of the 
generation of vorticity in premixed combustion. Louch \& Bray (1998) have performed two-dimensional simulations of Mueller's experiments.

Our aim is to investigate the effects mentioned above. For this we will make use of direct numerical simulation (DNS) of the flow field.

To reach our goal, the DNS should be able to reproduce the three aforementioned effects, i.e. distortion of the flame front, flame front instability and baroclinic vorticity production. The simulation of the first effect, distortion of the flame front, is straightforward, since in a DNS all turbulent length scales are resolved so that the deformation of the flame front can be computed directly. Instability of the flame front and baroclinic vorticity production are a result of gas expansion at the position of the flame front so that compressibility effects should be taken into account in our simulation. To accomplish this we consider the compressible Navier-Stokes equations in the low-Mach-number limit (Najm, Wyckoff \& Knio 1998). The advantage of this limit is that it allows us to use the efficient numerical methods, developed for incompressible flows. In previous studies, gas expansion has not been included or it has been included in such a way that it does not allow for baroclinic vorticity production. To our knowledge the study presented here is one of the first DNSs of a turbulent flow with premixed combustion which uses the level set approach and which includes all the aforementioned effects (e.g. in the review of Poinsot, Candel \& Trouvé (1996), no comparable simulations are mentioned). A comparison of the results obtained from our simulation with the results of other computations will thus allow for an investigation of the additional effects due to compressibility of the gas.

Since we consider premixed combustion in the flamelet regime, the actual combustion takes place in a very thin layer, the so-called flame front. A resolution of this flame front on the numerical grid, on which the flow is to be computed, would lead to inaccessible requirements in terms of computational power. Therefore we have chosen to model the flame front by means of a so-called level set approach (Sethian 1999). In this approach, the position of the flame front is represented by an isosurface $G_{0}$ of a scalar field $G(\boldsymbol{x}, t)$. The scalar field propagates with respect to the flow field with a flame velocity $s_{f}$. With the value of this flame velocity, all the internal structures of the flame region, which we cannot resolve on our numerical grid, are taken into account. The result is an equation which describes the evolution of the scalar field $G$ in time, the so-called $G$-equation, (Williams 1985a). The expansion of the gas is included by imposing a certain temperature and density profile at the position of the propagating flame. The size of the temperature jump over the flame front is determined by the properties of the chemical reaction and has to be known in advance. The same is true for the flame speed $s_{f}$, which is also assumed to be a known parameter.

The $G$-equation has been used previously in LES and DNS simulations. Im, Lund \& Ferziger (1995) and Piana, Veynante \& Candel (1996) studied the application of the $G$-equation in turbulent flow simulations to develop an LES-model (Im et al. 1997). Wenzel \& Peters (1998) did apply the $G$-equation in a DNS, but did not include the effect of gas expansion in their simulation. Much effort has been made to find a way to modify the $G$-equation itself to account for the effects of gas expansion so that one can continue to calculate with an incompressible flow. Ashurst (1997) has introduced a potential flow field which represents gas expansion as a source of volume. This potential flow field, however, lacks physical properties such as the generation of vorticity at the flame front. Nevertheless, Ashurst (1997) was able to reproduce the Darrieus-Landau instability. Peters, Wenzel \& Williams (2000) modified the $G$-equation in the Reynolds-averaged Navier-Stokes (RANS) equations to take a potential field into account. Wenzel \& Peters (2000) used a modified $G$-equation in a 
DNS model and concluded that the effects of gas expansion are only significant for small turbulence intensities.

Another model to compare with is presented by Zhang \& Rutland (1995). They modelled the chemical process with a single step reaction with a global reaction rate. This approach is, however, limited to low Reynolds numbers and relatively thick flames.

Our simulation is performed in a box geometry with two homogeneous directions. In the third direction, a realization of a stationary, homogeneous isotropic turbulent simulation is used as an inflow boundary condition with an uniform inflow velocity $U_{i n}$. In this geometry, regions of shear in the mean flow field, which usually occur in the vicinity of walls, are avoided. The disadvantage of such mean shear is that it influences the geometry of the flame front, which we want to avoid here since we are focusing on the influence of turbulence on the flame front. The turbulence decays while being transported downstream in through the box. The mean position of the flame front is stabilized at a fixed position by adjusting the inflow velocity $U_{i n}$. Such a stable position of the flame front gives us the ability to obtain accurate statistics by averaging in time together with averaging in the two homogeneous directions. Such time averaging is very difficult to achieve in experiments where the flame front usually moves.

In $\S 2$, we discuss the theoretical background of enhancement of premixed combustion by turbulence. In $\S 3$, the set of equations used in our simulations is presented and in $\S 4$, their numerical solution. The results of the simulations are discussed in $\S 5$.

\section{Theory}

We have argued that the propagation of the flame front by deflagration in premixed combustion is due to the diffusion of heat. Therefore, it follows that the speed of the flame front should depend on the diffusion coefficient of the gas and the combustion rate so that the speed of a laminar flame $s_{f l}$ can be expressed as (Williams 1985b):

$$
s_{f l} \approx \frac{1}{\rho_{u}} \sqrt{\frac{\lambda q}{c_{p}}},
$$

where $\rho_{u}$ is the density of the unburnt gas, $\lambda$ the thermal conductivity, $c_{p}$ the specific heat of the gas mixture and $q$ the chemical reaction rate or the amount of mass that is burnt per unit volume and time.

Let us now look in more detail at the structure of the flame (figure 1). We have already mentioned that the gas in front of the reaction region is heated by the diffusion until the ignition temperature, is reached. At the same time, the density will decrease and, as a consequence of continuity, the gas will expand so that the velocity will increase. When the gas reaches the ignition temperature, the gas will start to burn, as indicated in figure 1 by the chemical source term.

The flame with a total thickness $\ell_{f}$ consists of the preheat zone and the reaction zone. In the latter zone, the actual reaction takes place and its thickness, $\ell_{\delta}$, is of the order of $10 \%$ of the total flame thickness $\ell_{f}$ or

$$
\delta=\frac{\ell_{\delta}}{\ell_{f}} \approx 0.1 .
$$

When a flame front propagates through a turbulent flow field, its behaviour can be characterized by the ratio of the time scales of the turbulence and chemical processes 


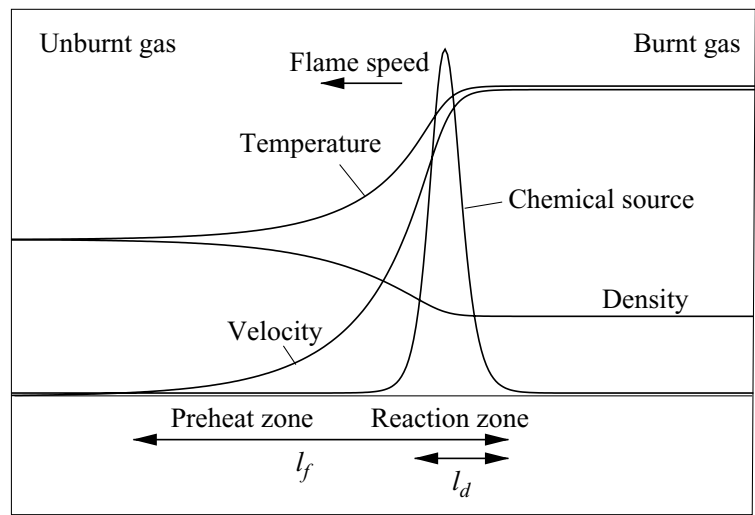

FIGURE 1. The structure of a stationary flame front in premixed combustion. (Note that in a frame fixed to the flame front, the product of density times velocity should be constant across the flame front.)

known as the Karlovitz number. Its definition is the ratio between the chemical time scale $\tau_{f}=\ell_{f} / s_{f l}$ and the Kolmogorov time scale $\tau_{\eta}$ :

$$
K a=\frac{\tau_{f}}{\tau_{\eta}}=\frac{\ell_{f}^{2}}{\eta^{2}},
$$

where $\eta$ is the Kolmogorov length scale.

For an extensive review on the various combustion regimes and their characteristics as a function of $K a$ we refer to literature (e.g. Peters 2000). Here, we shall limit ourselves to the case $K a<1$, known as the flamelet regime. In this regime, the inner structure of the flame will not be changed by the turbulent eddies, but the flame front will be deformed. The effects of the turbulence on the flame front are in this case purely kinematic and therefore ideal for modelling with the $G$-equation (Peters 2000).

\subsection{Turbulent distortion of the flame front}

Although for the flow regime that we have selected, turbulence is not able to influence the inner structure of the flame, turbulent eddies are able to deform the flame as a whole. Therefore, the flame front will become corrugated and wrinkled, which we denote as a kinematic interaction. The effect of this distortion is an increase in the total flame front area and therefore in the total combustion rate. Let us define a mean turbulent flame speed $s_{f t}$ by

$$
s_{f t}=s_{f l} \frac{A_{t}}{A_{l}},
$$

where $A_{t}$ is the total area of the turbulent flame front and $A_{l}$ the area of the flame front projected onto a flat surface, which would be the geometry of the flame in laminar flow conditions. Damköhler (1940) related the increase in flame area to the root mean square of the turbulent velocity fluctuations $u^{\prime}$ as follows:

$$
\frac{A_{t}}{A_{l}}=\frac{s_{f l}+C u^{\prime}}{s_{f l}},
$$

where $C$ is a coefficient with a value of order unity. Combined with (2.4), this gives:

$$
s_{f t}=s_{f l}+C u^{\prime},
$$




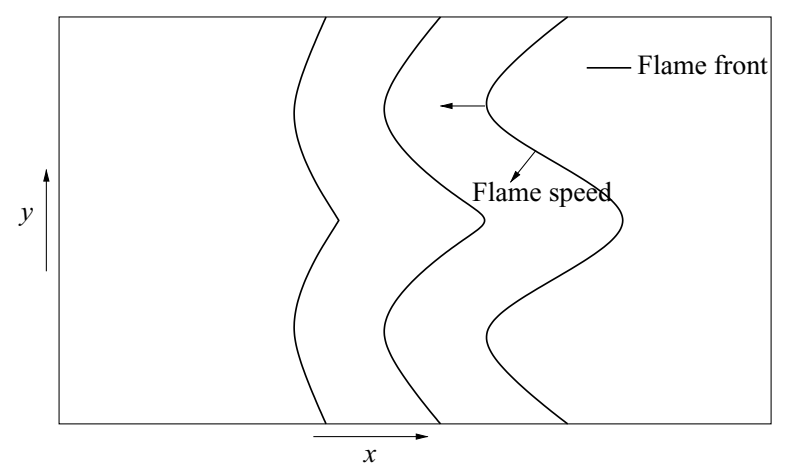

FigurE 2. Schematic illustration of the process of flame front restoration. The flame propagates from the right to the left, while the unburnt gas is at rest.

which suggests that the turbulent flame speed in the flamelet regime is linearly proportional to the turbulent velocity fluctuations.

The flame front propagates in the direction, normal to its surface and as a result it deforms the disturbances on the flame front into cusps, as shown in figure 2. These cusps move at a higher speed than the flame-front velocity. Therefore the amplitude of the disturbances are reduced and the flame front returns to its flat shape. This restoration of the flame front is called the Huygens effect in flame propagation, analogue to a similar effect which occurs in nonlinear optics.

\subsection{Flame front instabilities}

Next we turn to the so-called hydrodynamic instability, first considered by Landau (1944). The flame front is represented as a plane of discontinuity in the density. On this flame front a small-wavelike disturbance is superposed, given by

$$
\zeta=D \mathrm{e}^{\mathrm{i}(k y-\omega t),}
$$

where $k$ is the real wavenumber and $\omega$ the complex frequency. By linear stability theory, the following expression for the imaginary part of $\omega$, i.e. the growth rate of the amplitude of the disturbance, can be computed (Landau \& Lifshitz 2000, p. 488):

$$
\Omega=k u_{u} \frac{\tau+1}{\tau+2}\left(\sqrt{2+\tau-\frac{1}{\tau+1}}-1\right),
$$

where $u_{u}$ is the velocity of the unburnt gas and $\tau$ the ratio $\left(\rho_{u}-\rho_{b}\right) / \rho_{b}$. When $\tau>0$, which is the case for a flame, $\Omega>0$. This means that for all $k$, the disturbance on the flame front grows or the flame front is unconditionally unstable. This type of instability is usually denoted as the Darrieus-Landau instability.

For a further discussion on the background and mechanism of this instability see, e.g. Williams (1985b).

\section{The equations governing the premixed combustion process}

\subsection{The G-equation}

As mentioned before, the flame is modelled as an isosurface $G_{0}$ of a scalar field $G(\boldsymbol{x}, t)$ with $G_{0}(\boldsymbol{x}, t)$ indicating the position of the flame. The evolution of the scalar 
field $G$ is given by the equation:

$$
\frac{\partial G}{\partial t}+u_{j} \frac{\partial G}{\partial x_{j}}=s_{f}\left|\frac{\partial G}{\partial x_{i}}\right|,
$$

(Williams $1985 b$ ) which is known as the $G$-equation. The flame speed $s_{f}$ is the laminar flame speed with respect to the unburnt gas of a flat flame in a laminar flow $s_{f l}$, corrected for the effects of curvature and strain. It reads

$$
s_{f}=s_{f l}-s_{f l} \mathscr{L} \kappa-\mathscr{L} S .
$$

These corrections are valid only for low curvature and low strain limits. In (3.2) several parameters appear, which require an additional definition. The first is $\mathscr{L}$, the Markstein length. The second is the curvature $\kappa$, defined by

$$
\kappa=\nabla \cdot \boldsymbol{n}
$$

where the unit normal vector $\boldsymbol{n}$ can be written in terms of the $G$-field as

$$
\boldsymbol{n}=-\left.\frac{\nabla G}{|\nabla G|}\right|_{G=G_{0}} .
$$

Finally, $S$ is the strain rate imposed on the flame and it is given by

$$
S=-\boldsymbol{n} \cdot \nabla \boldsymbol{u} \cdot \boldsymbol{n} .
$$

The term with the curvature $\kappa$ acts as a diffusion term in the $G$-equation with a diffusion coefficient $\mathscr{D} \mathscr{L}$ given by

$$
\mathscr{D}_{\mathscr{L}}=s_{f l} \mathscr{L}
$$

The $\mathscr{D}_{\mathscr{L}}$ is called the Markstein diffusivity. According to Peters (2000), the Markstein length $\mathscr{L}$ and therefore $\mathscr{D}_{\mathscr{L}}$ is positive for most practical applications of premixed hydrocarbon combustion and has a value of the order of the laminar flame thickness $\ell_{f}$. For positive values of $\mathscr{L}$, the contribution of the curvature will suppress instabilities in the flame front. For negative values of $\mathscr{L}$ and $\mathscr{D} \mathscr{L}$, which occur in hydrogen-air mixtures, the instabilities will grow, leading to strong flame accelerations. In our computations, however, we have neglected all corrections on $s_{f l}$ by taking $\mathscr{L}=0$.

The flame speed must also be corrected for the effect of gas expansion. This correction can be written as (Piana et al. 1996)

$$
s_{f}=s_{f l} \frac{\rho_{u}}{\rho},
$$

so that the complete expression for $s_{f}$ becomes:

$$
s_{f}=\frac{\rho_{u}}{\rho}\left(s_{f l}-s_{f l} \mathscr{L} \kappa-\mathscr{L} S\right)
$$

This speed should be interpreted as the flame displacement speed. The heat release and the thermal expansion are, in principle, controlled by the flame consumption speed and these two velocities might be quite different (Poinsot Veynante \& Candel 1991; Piana et al. 1996; Poinsot \& Veynante 2001).

Besides the fact that the position of the flame front is represented by $G(x, t)=G_{0}$, the $G(\boldsymbol{x}, t)$ has no physical meaning. In order to simplify the coupling between $G$ and the temperature field, to be described in the next section, we impose the additional condition that $|\nabla G|=1$. This means that the value of $G(x, t)$ can be interpreted as the distance from $\boldsymbol{x}$ to the flame front. 


\subsection{The flow equations}

The flow is described by the compressible Navier-Stokes equations which read

$$
\frac{\partial \rho}{\partial t}+\frac{\partial m_{i}}{\partial x_{i}}=0
$$

and

$$
\frac{\partial m_{i}}{\partial t}+\frac{\partial m_{i} u_{j}}{\partial x_{j}}=-\frac{\partial p}{\partial x_{i}}+\frac{\partial \tau_{i j}}{\partial x_{j}}
$$

where $u_{i}$ is the velocity, $\rho$ is the density and $m_{i} \equiv \rho u_{i}$ the mass flux. The term $p$ is the pressure and $\tau_{i j}$ the deviatoric stress tensor. All variables have been made dimensionless with the laminar flame speed $s_{f l}$ as velocity scale and the height of our computational domain $L$ (which is equal to the width) as length scale. For the density and viscosity scale we take the values of the unburnt gas, i.e. $\rho_{u}$ and $\mu_{u}$. The Reynolds number $R e$ is then defined as:

$$
R e=\frac{\rho_{u} s_{f l} L}{\mu_{u}} .
$$

The stress tensor can be written as

$$
\tau_{i j}=\frac{1}{R e} \mu\left(\frac{\partial u_{i}}{\partial x_{j}}+\frac{\partial u_{j}}{\partial x_{i}}-\frac{2}{3} \delta_{i j} \Delta\right) .
$$

The term $\Delta$ is the relative change in volume, given by

$$
\Delta \equiv \frac{\partial u_{i}}{\partial x_{i}}=-\frac{1}{\rho} \frac{\mathrm{D} \rho}{\mathrm{D} t}
$$

where the expression on the far right-hand side follows from conservation of mass.

The dynamic viscosity $\mu$ is scaled with the unburnt gas $\mu_{u}$. In principle, the viscosity may depend on the temperature so that the viscosity may vary as a function of time and space. However, we assume here that the viscosity remains equal to the viscosity of the unburnt gas everywhere so that $\mu=1$. The stress gradient in (3.10) can now be written as

$$
\frac{\partial \tau_{i j}}{\partial x_{j}}=\frac{1}{R e}\left(\frac{\partial^{2} u_{i}}{\partial x_{j}^{2}}+\frac{1}{3} \frac{\partial \Delta}{\partial x_{i}}\right),
$$

In the low-Mach-number approximation (Najm et al. 1998), the pressure dependence of the density is neglected, resulting in the equation of state $\rho=\rho(T)$. Here we take for the medium an ideal gas and this leads to

$$
\rho=\frac{1}{T},
$$

where we have scaled the temperature $T$ with the temperature of the unburnt mixture $T_{u}$. With this form for the equation of state, all acoustic wave modes are eliminated from the system of equations, and efficient numerical methods, used in incompressible flows, can be applied.

Since $G$ is defined to be a distance function, the temperature of the gas can be derived directly from the $G$-field with

$$
T=T_{u}\left(1+\frac{\tanh \left(\left(G_{0}-G\right)\right) / \delta+1}{2} \tau\right),
$$


where $\tau$ is the heat release parameter defined in terms of the temperature or density of the unburnt mixture and the temperature $T_{b}$ or density $\rho_{b}$ of the burnt mixture as

$$
\tau=\frac{T_{b}-T_{u}}{T_{u}}=\frac{\rho_{u}-\rho_{b}}{\rho_{b}} .
$$

The $\delta$ in (3.16) should be interpreted as an 'artificial' thickness of the flame, which is required for numerical purposes. To resolve the flame in our computations $\delta$ is chosen such that the flame is about 4 grids cells wide. Because of computational limitations, the numerical flame thickness must be chosen to be larger than the flame thickness of a real flame. This artificial thickening of the flame will have some influence on the the local flame structure and topology. On the other hand, it will not have much influence on the global statistics and budgets of turbulence, because these quantities depend mainly on the large flow scales and on global conservation properties.

The $G$-equation (3.1), conservation of mass (3.9) and the momentum equation (3.10), combined with the equation of state (3.15) and the equation for the temperature (3.16) form a closed system which we will solve numerically.

\subsection{Turbulent kinetic energy}

In our analysis we shall consider the kinetic energy of the flow per unit volume which is defined as $k=\rho u_{i}^{2} / 2$. An equation for $k$ can be obtained by multiplying the momentum equation (3.10) with $u_{i}$, which leads to

$$
\frac{\partial k}{\partial t}+\frac{\partial u_{j} k}{\partial x_{j}}=-u_{i} \frac{\partial p}{\partial x_{i}}+u_{i} \frac{\partial \tau_{i j}}{\partial x_{j}} .
$$

In our case of a turbulent flow we will compute various flow statistics with the help of a Reynolds decomposition where each variable is written as the sum of a mean value and a fluctuation, i.e. $u=\bar{u}+u^{\prime}$. Here the overbar indicates an ensemble average and the prime indicates the fluctuation around this average. When we apply this procedure to the definition of the kinetic energy, we obtain the following expression for the mean kinetic energy $\bar{k}$,

$$
\bar{k}=\frac{1}{2} \bar{\rho} \bar{u}_{i}^{2}+\overline{\rho^{\prime} u_{i}^{\prime}} \bar{u}_{i}+\frac{1}{2} \bar{\rho} \overline{u_{i}^{\prime 2}}+\frac{1}{2} \overline{\rho^{\prime} u_{i}^{\prime 2}}
$$

which, because we have taken into account density fluctuations, becomes more complicated than the expression for $\bar{k}$ in incompressible flows. We must now make a distinction between the contribution to the kinetic energy by the mean flow and by the turbulence. We define the Galilean invariant part of (3.19) as the turbulent kinetic energy $k_{t}$ and the non-Galilean part of (3.19) as the mean flow kinetic energy $k_{m}$, so that $\bar{k}=\bar{k}_{m}+\bar{k}_{t}$. Both $k_{m}$ and $k_{t}$ can be divided into a mean and a fluctuating part which are given by:

$$
\begin{aligned}
& \bar{k}_{m}=\frac{1}{2} \bar{\rho} \bar{u}_{i}^{2}+\overline{\rho^{\prime} u_{i}^{\prime}} \bar{u}_{i}, \\
& k_{m}^{\prime}=\frac{1}{2} \rho^{\prime} \bar{u}_{i}{ }^{2}+\bar{\rho} \bar{u}_{i} u_{i}^{\prime}+\rho^{\prime} u_{i}^{\prime} \bar{u}_{i}-\overline{\rho^{\prime} u_{i}^{\prime}} \bar{u}_{i},
\end{aligned}
$$

and

$$
\begin{aligned}
& \bar{k}_{t}=\frac{1}{2} \bar{\rho} \overline{u_{i}^{\prime 2}}+\frac{1}{2} \overline{\rho^{\prime} u_{i}^{\prime 2}}, \\
& k_{t}^{\prime}=\frac{1}{2} \bar{\rho} u_{i}^{\prime 2}-\frac{1}{2} \bar{\rho} \overline{u_{i}^{\prime 2}}+\frac{1}{2} \rho^{\prime} u_{i}^{\prime 2}-\frac{1}{2} \overline{\rho^{\prime} u_{i}^{\prime 2}},
\end{aligned}
$$


An equation for $\bar{k}_{m}$ can be derived by applying a Reynolds decomposition to the momentum equation (3.10) and multiplying the mean part with $\bar{u}_{i}$. This results in:

$$
\begin{aligned}
\frac{\partial \bar{k}_{m}}{\partial t}+\frac{\partial \bar{u}_{j} \bar{k}_{m}}{\partial x_{j}}= & -\frac{\partial \overline{u_{j}^{\prime} k_{m}^{\prime}}}{\partial x_{j}}-\bar{u}_{i} \frac{\partial \bar{p}}{\partial x_{i}}+\bar{u}_{i} \frac{\partial \bar{\tau}_{i j}}{\partial x_{j}}+\overline{\rho^{\prime} u_{i}^{\prime}} \frac{\partial \bar{u}_{i}}{\partial t} \\
& +\bar{\rho} \overline{u_{i}^{\prime} u_{j}^{\prime}} \frac{\partial \bar{u}_{i}}{\partial x_{j}}+\overline{\rho^{\prime} u_{i}^{\prime}} \bar{u}_{j} \frac{\partial \bar{u}_{i}}{\partial x_{j}}+\overline{\rho^{\prime} u_{i}^{\prime} u_{j}^{\prime}} \frac{\partial \bar{u}_{i}}{\partial x_{j}}
\end{aligned}
$$

An equation for $\bar{k}_{t}$ can be found by taking the fluctuating part of the momentum equation, multiplying this with $u_{i}^{\prime}$ and averaging the result. This gives:

$$
\begin{aligned}
\frac{\partial \bar{k}_{t}}{\partial t}+\frac{\partial \bar{u}_{j} \bar{k}_{t}}{\partial x_{j}}= & -\frac{\partial \overline{u_{j}^{\prime} k_{t}^{\prime}}}{\partial x_{j}}-\overline{u_{i}^{\prime} \frac{\partial p^{\prime}}{\partial x_{i}}}+\overline{u_{i}^{\prime} \frac{\partial \tau_{i j}^{\prime}}{\partial x_{j}}} \\
& -\left(\overline{\rho^{\prime} u_{i}^{\prime}} \frac{\partial \bar{u}_{i}}{\partial t}+\bar{\rho} \overline{u_{i}^{\prime} u_{j}^{\prime}} \frac{\partial \bar{u}_{i}}{\partial x_{j}}+\overline{\rho^{\prime} u_{i}^{\prime}} \bar{u}_{j} \frac{\partial \bar{u}_{i}}{\partial x_{j}}+\overline{\rho^{\prime} u_{i}^{\prime} u_{j}^{\prime}} \frac{\partial \bar{u}_{i}}{\partial x_{j}}\right) .
\end{aligned}
$$

Substitution of the expression for the deviatoric stress (3.14) gives

$$
\overline{u_{i}^{\prime} \frac{\partial \tau_{i j}^{\prime}}{\partial x_{j}}}=\frac{1}{R e}\left(\frac{\partial^{2} \overline{\frac{1}{2} u_{i}^{\prime 2}}}{\partial x_{j}^{2}}-\overline{\left(\frac{\partial u_{i}^{\prime}}{\partial x_{j}}\right)^{2}}+\frac{\partial \overline{\frac{1}{3} u_{i}^{\prime} \Delta^{\prime}}}{\partial x_{i}}-\frac{1}{3} \overline{\Delta^{\prime 2}}\right) .
$$

This, together with the assumption that the problem is stationary, gives the following equation for the turbulent kinetic energy budget:

$$
\frac{\mathrm{D} \bar{k}_{t}}{\mathrm{D} t} \equiv \bar{u}_{j} \frac{\partial \bar{k}_{t}}{\partial x_{j}}=E_{k}+T_{k}+P_{k}+\Pi_{k}+D_{k}+\epsilon_{k}=0,
$$

with

$$
\begin{aligned}
E_{k} & =-\bar{k}_{t} \frac{\partial \bar{u}_{i}}{\partial x_{i}} \\
T_{k} & =-\frac{\partial \overline{u_{j}^{\prime} k_{t}^{\prime}}}{\partial x_{j}}, \\
P_{k} & =-\left(\bar{\rho} \overline{u_{i}^{\prime} u_{j}^{\prime}} \frac{\partial \bar{u}_{i}}{\partial x_{j}}+\overline{\rho^{\prime} u_{i}^{\prime}} \bar{u}_{j} \frac{\partial \bar{u}_{i}}{\partial x_{j}}+\overline{\rho^{\prime} u_{i}^{\prime} u_{j}^{\prime}} \frac{\partial \bar{u}_{i}}{\partial x_{j}}\right), \\
\Pi_{k} & =-\frac{\partial \overline{p^{\prime} u_{i}^{\prime}}}{\partial x_{i}}+\overline{p^{\prime}} \frac{\partial u_{i}^{\prime}}{\partial x_{i}} \\
D_{k} & =\frac{1}{R e}\left(\frac{\partial^{2} \frac{1}{2} u_{i}^{\prime 2}}{\partial x_{j}^{2}}+\frac{\partial \frac{1}{3} u_{i}^{\prime} \Delta^{\prime}}{\partial x_{i}}\right) \\
\epsilon_{k} & =-\frac{1}{R e}\left(\overline{\left(\frac{\partial u_{i}^{\prime}}{\partial x_{j}}\right)^{2}}+\overline{\frac{1}{3} \Delta^{\prime 2}}\right) .
\end{aligned}
$$

Some of the terms in (3.27) have a similar interpretation as in the incompressible case. These are the production term $P_{k}$, the turbulent transport term $T_{k}$, the viscous and pressure transport $D_{k}$ and the viscous dissipation $\epsilon_{k}$. However, all these terms are more complicated than in the incompressible case, because of the inclusion of density fluctuations. This is in particular apparent in the production term, which contains now three terms instead of one term. However, the interpretation of the production 
term as the interaction between the mean flow and the turbulence remains, because this term also appears in the equation for $\bar{k}_{m}$ with an opposite sign.

There appear also new terms in (3.27) which are not found in the equation for the incompressible turbulent kinetic energy. The first is $E_{k}$, which can be interpreted as the effect of volume expansion on the turbulent kinetic energy. In our case where the gas expands, i.e. $\partial u_{i} / \partial x_{i}>0$, this term results in a decrease of the kinetic energy. A simple explanation is that in the case of gas expansion, the volume increase results in a decrease of the energy density. The pressure term $\Pi_{k}$ consists of a contribution of the pressure diffusion, which also occurs in the incompressible energy budget, and a new contribution, which is called the pressure dilation. The pressure dilation describes the contribution to the kinetic energy as a result of the expansion work by the pressure field.

\subsection{Vorticity}

In our further analysis we will also consider the vorticity, in particular in connection with vorticity production by baroclinic terms. By applying the rotational operator to (3.10) we obtain the following equation for the vorticity:

$$
\frac{\partial \boldsymbol{\omega}}{\partial t}+\boldsymbol{u} \cdot \nabla \boldsymbol{\omega}=(\boldsymbol{\omega} \cdot \nabla) \boldsymbol{u}-\boldsymbol{\omega}(\nabla \cdot \boldsymbol{u})-\left(\nabla \frac{1}{\rho} \times \nabla p\right)+\frac{1}{\operatorname{Re}} \frac{1}{\rho} \nabla^{2} \boldsymbol{\omega}+\left(\nabla \frac{1}{\rho}\right) \times(\nabla \cdot \boldsymbol{\tau}) .
$$

Let us first consider the terms in this equation which also appear in the vorticity equation for an incompressible flow (Batchelor 1967, §5.2). Apart from the terms on the left-hand side of the equation, the first term on the right-hand side $(\omega \cdot \nabla) \boldsymbol{u}$, describes the effect of deformation of the velocity field on the vorticity. One of the consequences of this term is that it causes a vortex to spin up, when it is stretched in the direction of its vorticity vector. The second term is $\nabla^{2} \boldsymbol{\omega}$ and it accounts for the viscous destruction of vorticity.

Next we consider the terms which do not have an equivalent term in the incompressible vorticity equation. This is the term $-\boldsymbol{\omega}(\nabla \cdot u)$, which describes the effect of gas expansion. When the gas expands $\omega$ will decrease since it is smeared out over a larger volume. The term $-\nabla(1 / \rho) \times \nabla p$ is called the baroclinic production term and it describes the production of vorticity when the pressure and density gradient are not aligned. For the same pressure gradient, the part of the flow with a low density will be accelerated faster than the part with the higher density, resulting in the production of vorticity. The last term, which is written as $\nabla(1 / \rho) \times(\nabla \cdot \tau)$, is similar to the baroclinic term in the way that it accounts for the production of vorticity when the density gradient and the divergence of the stress are not aligned.

In our analysis we will be primarily interested in the vorticity fluctuations. A measure of these fluctuations is the enstrophy $\Omega$, which is defined as

$$
\Omega=\overline{\frac{1}{2}\left|\omega^{\prime}\right|^{2}},
$$

where $\omega^{\prime}$ is the vorticity fluctuation around the average vorticity $\overline{\boldsymbol{\omega}}$. An expression for $\Omega$ can be found by a similar procedure as used to obtain the equation for the turbulent kinetic energy. It is convenient to write the resulting equation with the help of the specific volume $v=1 / \rho$ and the quantity $\Omega^{\prime}$, defined as

$$
\Omega^{\prime} \equiv \frac{1}{2}\left|\omega^{\prime}\right|^{2}=\frac{1}{2} \omega^{\prime} \cdot \omega^{\prime}
$$

The resulting equation for the enstrophy then becomes:

$$
\frac{\partial \Omega}{\partial t}+\overline{\boldsymbol{u}} \cdot \nabla \Omega=E_{\omega}+T_{\omega}+\Pi_{\omega}+D_{\omega}+S_{\omega}+\epsilon_{\omega}
$$


with

$$
\begin{aligned}
E_{\omega} & =-\Omega(\nabla \cdot \bar{u}), \\
T_{\omega} & =-\nabla \cdot \overline{\left(\boldsymbol{u}^{\prime} \Omega^{\prime}\right)}, \\
\Pi_{\omega} & =-\overline{\omega^{\prime} \cdot\left(\nabla v^{\prime} \times \nabla p^{\prime}\right)}+\overline{\omega^{\prime} \cdot\left(\nabla v^{\prime} \times \nabla \cdot \tau^{\prime}\right)}, \\
D_{\omega} & =\frac{1}{\operatorname{Re}} \bar{v} \nabla^{2} \Omega+\frac{1}{\operatorname{Re}} \overline{v^{\prime} \nabla^{2} \Omega^{\prime}}, \\
S_{\omega} & =\overline{\omega^{\prime} \cdot\left(\omega^{\prime} \cdot \nabla \overline{\boldsymbol{u}}\right)}+\overline{\omega^{\prime} \cdot\left(\omega^{\prime} \cdot \nabla \boldsymbol{u}^{\prime}\right)}, \\
\epsilon_{\omega} & =-\frac{1}{\operatorname{Re}} \bar{v} \overline{\left\|\nabla \omega^{\prime}\right\|^{2}}+\frac{1}{\operatorname{Re} \overline{v^{\prime}\left\|\nabla \omega^{\prime}\right\|^{2}} .}
\end{aligned}
$$

The $E_{\omega}$ denotes the effect of the expansion on the enstrophy. This term has the same effect as the equivalent term in the equation of kinetic energy and will lower the enstrophy density, due to the increase in volume in an expanding flow. The $T_{\omega}$ is the turbulent transport term and $\Pi_{\omega}$ is the production of enstrophy, as the result of the normal and deviatoric stresses acting on a fluid with a density gradient. The first of these terms is the baroclinic production of enstrophy. The $D_{\omega}$ is the viscous transport term, $P_{\omega}$ is the production of enstrophy by the deformation of the velocity field and $\epsilon_{\omega}$ is the viscous destruction of enstrophy.

\subsection{Favre averaging}

The complication that we have encountered in $\S \S 3.3$ and 3.4 because of the appearance of density fluctuations, is usually avoided by introducing a so-called Favre averaging (Lele 1994), defined as

$$
\widetilde{u}_{i}=\frac{\overline{\rho u_{i}}}{\bar{\rho}} .
$$

When we, for instance, substitute this definition into (3.10) we obtain the following equation for the Favre mean velocity

$$
\frac{\partial \bar{\rho} \widetilde{u}_{i}}{\partial t}+\frac{\partial \bar{\rho} \widetilde{u}_{i} \widetilde{u}_{j}}{\partial x_{j}}=-\frac{\partial \bar{p}}{\partial x_{i}}+\frac{\partial \bar{\tau}_{i j}}{\partial x_{j}}-\frac{\partial \bar{\rho} \widetilde{u_{i}^{\prime \prime} u_{j}^{\prime \prime}}}{\partial x_{j}},
$$

where the velocity fluctuation $u_{i}^{\prime \prime}$ is defined by $u_{i}^{\prime \prime}=u_{i}-\widetilde{u}_{i}$.

The advantage of this description is that the equation (3.33) has the same form as the equation for the mean velocity for an incompressible flow. This is also the case for the Favre-averaged kinetic energy, which is defined as

$$
\widetilde{k}=\widetilde{k}_{m}+\widetilde{k}_{t}=\frac{1}{2} \widetilde{\rho} \widetilde{u}_{i}^{2}+\frac{1}{2} \widetilde{\rho} \widetilde{u_{i}^{\prime \prime 2}} .
$$

The $\widetilde{k}_{t}$ can be expressed in terms of the variables that we use here:

$$
\widetilde{k}_{t}=\frac{1}{2}\left(\bar{\rho} \overline{u_{i}^{\prime 2}}+\overline{\rho^{\prime} u_{i}^{\prime 2}}-\frac{{\overline{\rho^{\prime} u_{i}^{\prime}}}_{\bar{\rho}}^{2}}{\bar{\rho}}\right)=\bar{k}_{t}-\frac{1}{2} \frac{{\overline{\rho^{\prime} u_{i}^{\prime}}}^{2}}{\bar{\rho}} .
$$

We see thus that the Favre-averaged turbulent kinetic energy will always be smaller than the ensemble mean turbulent kinetic energy given by (3.22). We shall return to this result in our analysis of the simulation data.

\section{Numerical method}

To compute the problem described by the equations (3.1), (3.9) and (3.10), we use a second-order predictor-corrector projection scheme (Najm et al. 1998) on a staggered 
grid. In this scheme, the evolution of the velocity and $G$-equation are coupled in order to stabilize the computation which in our case concerns a flow with large density variations. The spatial derivatives in equations (3.9) and (3.10) are obtained with a finite-volume method on a staggered grid, resulting in a second-order accurate discretization. The disadvantage of the low-order numerical methods, such as the one used here, is that they are always affected by some numerical dissipation (or numerical viscosity). Their advantage, however, is that these methods have certain nice properties, such as conservation of kinetic energy, which most high-order methods do not have.

The spatial derivatives in equation (3.1) are also obtained with a finite-volume method on a staggered grid. The advective fluxes on the cell faces of the control volumes are obtained with the help of a TVD-scheme with a Van Leer flux limiter.

\subsection{Predictor}

The predicted values at the new time are denoted with an asterisk.

First, we integrate the G-equation (3.1), in time using a second-order AdamsBashforth scheme to obtain a prediction of $G$ according to

$$
\frac{G^{*}-G^{n}}{\Delta t}=\left.\frac{3}{2} \frac{\partial G}{\partial t}\right|^{n}-\left.\frac{1}{2} \frac{\partial G}{\partial t}\right|^{n-1},
$$

where $\partial G /\left.\partial t\right|^{n}$ is discretized on the spatial grid using a finite-volume method. After this step, $G$ is reinitialized with the procedure of Sussman, Smereka \& Osher (1994), in order to satisfy the condition that $|\nabla G|=1$ everywhere. From the value of $G^{*}$, we obtain the temperature by means of (3.16) and from the temperature we calculate the density $\rho^{*}$ with the help of (3.15).

The flow field is now solved by means of a pressure-correction method. We integrate the momentum equations (3.10), again with a second-order Adams-Bashforth scheme,

$$
\frac{\boldsymbol{m}^{\prime}-\boldsymbol{m}^{n}}{\Delta t}=\left.\frac{3}{2} \frac{\partial \boldsymbol{m}}{\partial t}\right|^{n}-\left.\frac{1}{2} \frac{\partial \boldsymbol{m}}{\partial t}\right|^{n-1},
$$

where $\boldsymbol{m}^{\prime}$ is called the intermediate mass flux. The time derivatives on the right-hand side of (4.2) are defined as

$$
\left.\frac{\partial \boldsymbol{m}}{\partial t}\right|^{n}=-\nabla \cdot\left(\boldsymbol{m}^{n} \boldsymbol{u}^{n}+\boldsymbol{\tau}^{n}\right),
$$

where spatial derivatives are discretized with help of a second-order central scheme.

Conservation of mass is enforced by introducing the pressure in

$$
\frac{\boldsymbol{m}^{*}-\boldsymbol{m}^{n}}{\Delta t}+\nabla \cdot\left(\boldsymbol{m}^{n} \boldsymbol{u}^{n}\right)=-\nabla p^{*}+\nabla \cdot \boldsymbol{\tau}^{n},
$$

with $\boldsymbol{m}^{*}$ again the predicted mass flux. Subtracting both expressions (4.2) and (4.4), gives

$$
\frac{\boldsymbol{m}^{*}-\boldsymbol{m}^{\prime}}{\Delta t}=-\nabla p^{*},
$$

Application of conservation of mass (3.9) gives

$$
\nabla^{2} p^{*} \Delta t=\nabla \cdot \boldsymbol{m}^{\prime}-\left.\frac{\partial \rho}{\partial t}\right|^{*},
$$


which has the form of a Poisson equation for the pressure. The term $\partial \rho /\left.\partial t\right|^{*}$ cannot be evaluated directly and is obtained from the following second-order discretization:

$$
\left.\frac{\partial \rho}{\partial t}\right|^{*}=\frac{1}{2 \Delta t}\left(3 \rho^{*}-4 \rho^{n}+\rho^{n-1}\right) .
$$

The equation (4.6) is solved with a direct solver using Fourier transforms in the periodic $y$ - and $z$-directions. For the $x$-direction, a tridiagonal system remains, which can be solved directly. With the pressure determined, we correct the intermediate mass flux with the help of (4.5) which gives us a prediction of the mass flux $\boldsymbol{m}^{*}$ on the new time. The velocity is computed from this mass flux and the predicted density.

The method described above is the standard projection method, as applied in the case of incompressible flows. It is known to be stable for temperature and density ratios up to 2 so that in those cases the values of $G^{*}, m^{*}, u^{*}$ and $\rho^{*}$ can be taken equal to the values $G^{n+1}, m^{n+1}, u^{n+1}$ and $\rho^{n+1}$ at the next time step. For higher density ratios, a corrector step is required to stabilize the scheme.

\subsection{Corrector}

The predicted values, denoted with an asterisk, are now corrected to obtain the final value at the new time. The temporal derivative of $G$ on the new time level $n+1$ is estimated with the help of the predicted values indicated with an asterik, with the help of a quasi-implicit Crank Nicolson scheme:

$$
\frac{G^{n+1}-G^{n}}{\Delta t}=\frac{1}{2}\left(\left.\frac{\partial G}{\partial t}\right|^{n}+\left.\frac{\partial G}{\partial t}\right|^{*}\right)
$$

After this, the reinitialization procedure is applied again to obtain $\left|\nabla G^{n+1}\right|=1$ everywhere. The new density $\rho^{n+1}$ is again found from the equation for the temperature (3.16) and the equation of state (3.15). The new intermediate mass flux $\boldsymbol{m}^{\prime \prime}$ is now given by

$$
\frac{\boldsymbol{m}^{\prime \prime}-\boldsymbol{m}^{n}}{\Delta t}=\frac{1}{2}\left(\left.\frac{\partial \boldsymbol{m}}{\partial t}\right|^{n}+\left.\frac{\partial \boldsymbol{m}}{\partial t}\right|^{*}\right)
$$

where the estimate of the temporal derivative on the new time step is based on the discretization of the predicted mass flux and velocity field according to

$$
\left.\frac{\partial \boldsymbol{m}}{\partial t}\right|^{*}=-\nabla \cdot\left(\boldsymbol{m}^{*} \boldsymbol{u}^{*}+\boldsymbol{\tau}^{*}\right)
$$

The pressure is then solved, using density on the new time step,

$$
\nabla^{2} p^{n+1} \Delta t=\nabla \cdot \boldsymbol{m}^{\prime \prime}-\left.\frac{\partial \rho}{\partial t}\right|^{n+1}
$$

where

$$
\left.\frac{\partial \rho}{\partial t}\right|^{n+1}=\frac{1}{2 \Delta t}\left(3 \rho^{n+1}-4 \rho^{n}+\rho^{n-1}\right) .
$$

With this pressure, the mass flux is corrected according to

$$
\frac{\boldsymbol{m}^{n+1}-\boldsymbol{m}^{\prime \prime}}{\Delta t}=-\nabla p^{n+1}
$$

At this point, all variables at the time $n+1$ are known and the computation can be continued for the next time step. 


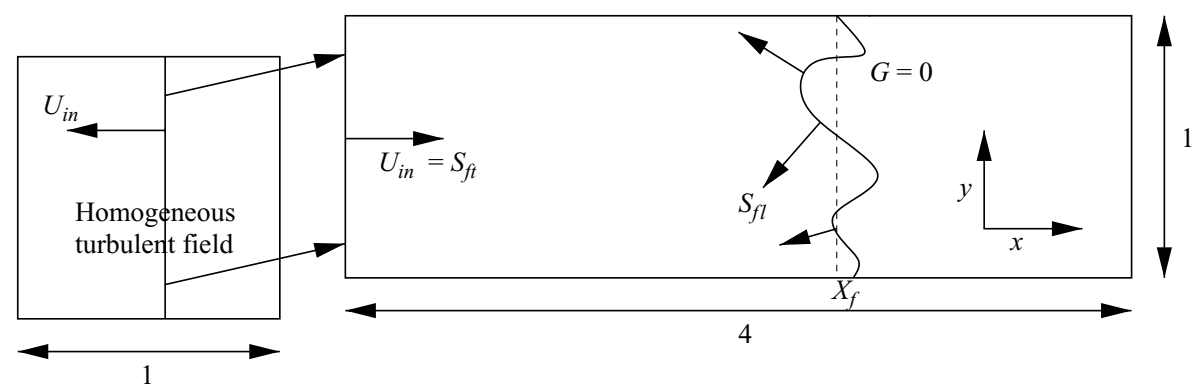

FIGURE 3. Sketch of the flow geometry.

\section{Computations}

\subsection{Computational domain}

The computational geometry is a box with sides $L_{x}, L_{y}$ and $L_{z}$, with $L_{y}=L_{z}$ and $L_{x}=4 L_{y}$. A sketch of the geometry is given in figure 3 . The mean flow is from left to right, while the flame front propagates in the opposite direction.

As inflow condition at $x=0$ we have used a realization of homogeneous isotropic turbulence in a periodic box, computed with a resolution of $128^{3}$. This isotropic turbulence field is fed into the channel at $x=0$ with a inflow velocity $U_{i n}$ constant over the cross-section of the channel. Furthermore, the inflow velocity is adjusted at every time step to compensate for the turbulent flame speed $s_{f t}$ so that the flame stays at approximately the same position in the box. The adjustment procedure is described in the following.

The position of the flame front $x_{f}$ is defined as

$$
x_{f}=\frac{\int_{V}(x-G) \mathrm{d} V}{V},
$$

where $V$ is the total flow volume. This flame position is stabilized around the desired position $x_{0}$ by adjusting the inflow velocity $U_{\text {in }}$ according to

$$
\frac{\mathrm{d} U_{i n}}{\mathrm{~d} t}=-c_{1}\left(x_{f}-x_{0}\right)-c_{2} \frac{\mathrm{d} x_{f}}{\mathrm{~d} t} .
$$

For the position of the flame front we can write

$$
x_{f}-x_{0}=\int_{0}^{t}\left[U_{i n}\left(t^{\prime}\right)-s_{f}\left(x^{\prime}\right)\right] \mathrm{d} t^{\prime},
$$

where we have assumed that the flame front starts at $x_{0}$ for $t=0$ and that $s_{f}$ depends on time through its position. After differentiating this relation twice and the substitution of (5.2), we find that $x_{f}$ satisfies the equation

$$
\frac{\mathrm{d}^{2} x_{f}}{\mathrm{~d} t^{2}}=-c_{1}\left(x_{f}-x_{0}\right)-\left(c_{2}+\frac{\mathrm{d} s_{f}}{\mathrm{~d} x}\right) \frac{\mathrm{d} x_{f}}{\mathrm{~d} t} .
$$

The first term in this equation give rise to a harmonic oscillation of $x_{f}$ around $x_{0}$ while the second term presents a damping when $c_{2}+\mathrm{d} s_{f} / \mathrm{d} x>0$. We note here that we have argued, e.g. in (2.5), that $s_{f}$ will be a funtion of $u^{\prime}$. In the flows that we consider here, $\mathrm{d} u^{\prime} / \mathrm{d} x<0$ so that also $\mathrm{d} s_{f} / \mathrm{d} x<0$. This means that without a sufficiently large 
positive $c_{2}$ our system would be unstable. In our case we try to have a behaviour of $x_{f}$ close to critically damped for which the relation $c_{2}+\mathrm{d} s_{f} / \mathrm{d} x=2 \sqrt{c_{1}}$ should be satisfied. This means that the damping is strong enough to avoid unnecessary oscillations around $x_{0}$, but not so strong as to avoid a too quick return to equilibrium. The constant $c_{1}$ can be chosen freely and determines the stiffness of the system. In this case, we take $c_{1}=10$. With this value, the position of the flame is fairly stable and varies no more than $2 \%$ around its equilibrium position, while it gives no additional limitation of the time step.

Four types of simulations, called types I, II, III and IV, have been performed. In the first type we consider spatially decaying incompressible turbulence. The resolution of the type I simulation is $384 \times 128 \times 128$ in the $x$-, $y$ - and $z$-directions, respectively. The grid spacing is chosen uniform in all directions, which minimizes the numerical dissipation of the second-order scheme that we use. This simulation can be considered as a reference for decaying turbulence when no combustion effects are present. The inflow velocity at $x=0$ in this case is fixed to $U_{\text {in }}=1$.

The second type of simulation is performed with an imposed gas expansion with $\tau=3$ at a fixed position at $x=2.66$. The flame is kept flat which implies that flame distortion by turbulence and instability processes is turned off. As a result of the fixed position of the flame front, the inflow velocity $U_{\text {in }}$ need not be adjusted to stabilize the flame front, but it remains constant with a value of $U_{i n}=1$. This case is not a realistic combustion simulation, since the flame front is not affected by the turbulence, but remains flat; however, the turbulence is affected by the expansion of the gas. This simulation is denoted the 'flat flame' in the graphs.

The third type of simulation is performed to investigate whether the code is able to reproduce the Darrieus-Landau instability of the flame front. This can be considered a test of our code to check whether compressibility effects have been correctly incorporated. The simulations are performed on a two-dimensional geometry and are carried out by using our code with a minimal number of grid points in the $z$-direction. We have used a resolution of $256 \times 128 \times 4$ on a domain of $2 \times 1 \times 0.03125$ in the $x$-, $y$ - and $z$-directions. These tests are performed on a PC with a $900 \mathrm{MHz}$ Athlon CPU. Each time step takes about $3 \mathrm{~s}$ and a run takes 1000 time steps.

In the fourth type of simulation, we carry out the full DNS of a turbulent flow including the interaction between the turbulence and the flame front. These simulations use the same turbulent inflow boundary conditions, as mentioned in the type I simulations. The simulations are performed on a grid in the domain shown in figure 3 with a resolution of $384 \times 128 \times 128$ on an SGI O3800 computer. The code is parallelized and runs on 32 nodes. Each time step takes about 5 wall clock CPU s. A run takes about 80000 time steps.

The statistical results to be presented below are obtained by taking an ensemble average over at least 50 realizations of the flow field. In order to eliminate the effect of the flame-front stabilization, mentioned above, on the statistics we compute the fluctuating quantities with respect to the mean of each realization and not, as is often done, with respect to the total mean. This has the advantage that the fluctuations in $U_{i n}$, which result from the stabilization mechanism, and the fluctuations in the pressure gradient, which result from the change in the bulk velocity, give no contribution to the statistics of the velocity fluctuations and the pressure.

The realization of homogeneous isotropic turbulence, which is applied as inflow boundary condition for the type I, II and IV simulations, is obtained from a pseudospectral code used by Brethouwer (2001). This simulation, which we will call the inflow field, has a Reynolds number of $R e_{\lambda}=95$, where $R e_{\lambda}$ is defined in terms of the 


\begin{tabular}{|c|c|c|c|c|c|c|c|}
\hline Type & $\begin{array}{l}\text { Heat release } \\
\text { included }\end{array}$ & $\begin{array}{c}\text { Heat release } \\
\text { parameter } \tau \\
\text { at } x=0\end{array}$ & $\begin{array}{l}\text { Turbulence } \\
\text { intensity } v^{\prime}\end{array}$ & $\begin{array}{l}\text { Reynolds } \\
\text { number } \\
R e_{\ell}\end{array}$ & $\begin{array}{c}\text { Inflow } \\
\text { velocity } U_{i n}\end{array}$ & $\begin{array}{l}\text { Position } \\
\text { of flame } \\
\overline{x_{f}}\end{array}$ & $\begin{array}{l}\text { Indicated } \\
\text { with }\end{array}$ \\
\hline I & No & 0 & 2.35 & 818 & 1 (fixed) & & $\tau=0, U_{i n}=1$ \\
\hline II & Yes & 3 & 2.35 & 818 & 1 (fixed) & 2.66 & flat flame \\
\hline IV & No & 0 & 0.59 & 205 & 1.28 & 2.70 & \\
\hline IV & No & 0 & 1.17 & 409 & 1.34 & 2.69 & \\
\hline IV & No & 0 & 2.35 & 818 & 1.41 & 2.69 & $\tau=0$ \\
\hline IV & Yes & 1 & 2.35 & 818 & 1.88 & 2.62 & $\tau=1$ \\
\hline IV & Yes & 3 & 2.35 & 818 & 3.38 & 2.51 & $\tau=3$ \\
\hline IV & Yes & 5 & 2.35 & 818 & 4.44 & 2.59 & $\tau=5$ \\
\hline
\end{tabular}

TABLE 1. Overview of the different types of three-dimensional simulations.

r.m.s. velocity fluctuation by

$$
v^{\prime}=\sqrt{\frac{\overline{u_{i}^{2}}}{3}},
$$

and the Taylor microscale $\lambda$ as

$$
\operatorname{Re}_{\lambda}=\frac{v^{\prime} \lambda}{v}
$$

In this case, $\lambda=0.0518 L$ and the integral length scale $\ell=0.174 L$, where $L$ is the length of the side of the cube, in which the simulation of the homogeneous isotropic turbulent flow field is carried out. The Reynolds number, based on $\ell$ and $v^{\prime}$ according to,

$$
R e_{\ell}=\frac{v^{\prime} \ell}{v}=\frac{\ell}{\lambda} R e_{\lambda}
$$

becomes $R e_{\ell}=319$. From these data, we can compute the viscosity of the inflow field in order to set the correct parameters for the simulation of the combustion.

An overview of all the simulations of types I, II and IV that we have carried out, is shown in table 1 . We see that various values for the inflow Reynolds number $R e_{\ell}$ have been used. For instance, all the simulation with $v^{\prime}=2.35$ have a Reynolds number of $R e_{\ell}=818$, which means that the viscosity in the simulation is a factor 2.56 lower than the viscosity in the inflow field, which has a Reynolds number of $R e_{\ell}=319$. For $v^{\prime}=2.35$, the viscosity is adjusted in order to lower the decay rate of the turbulence, which results in higher turbulence intensities at the position of the flame front. For the cases $v^{\prime}=1.17$ and $v^{\prime}=0.59$, the velocity of the inflow field is scaled with a factor of 0.5 and 0.25 , respectively, with respect to the case $v^{\prime}=2.35$, while the viscosity is kept constant. This results in values of the Reynolds number of $R e_{\ell}=409$ and $R e_{\ell}=205$, as listed in table 1 . A disadvantage of this approach is that the viscosity of the inflow field and the channel are not equal and the flow field in the channel has to adjust to the different viscosity. However, owing to the artificial nature of the inflow boundary condition, the flow in the channel must adjust itself anyway, even when the viscosity of the inflow field and the channel match exactly. The effect of this flow adjustment can be seen in figures 14 and 19 where we show the decay of turbulent kinetic energy and the decay of the enstrophy, respectively. For $\tau=3$ and $\tau=5$, the decay of the turbulent kinetic energy is relatively low from $x=0$ to $x=0.5$, while in the same region and for the same values of $\tau$, the enstrophy even shows an increase. However after $x=0.5$, the flow behaves as a fully developed decaying turbulent flow 


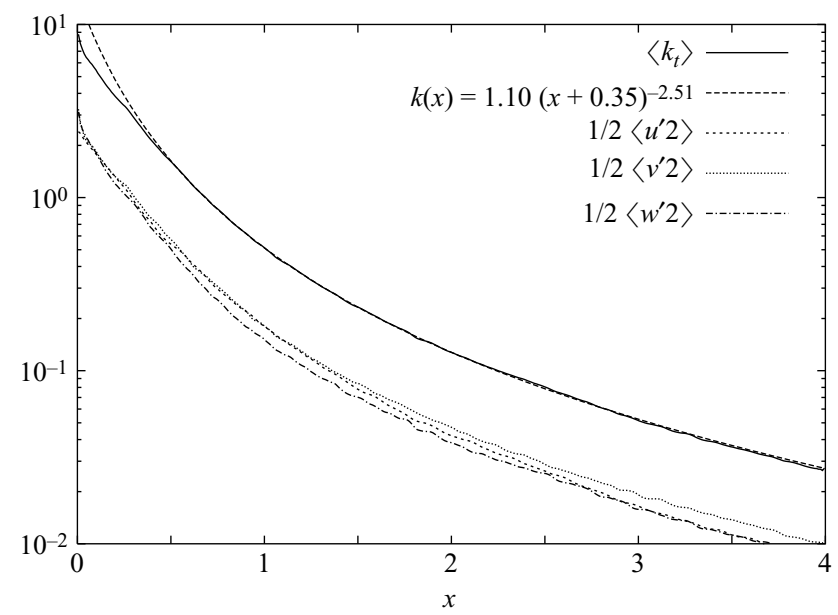

FIGURE 4. The turbulent kinetic energy $k_{t}$ and its components as a function of $x$. The total energy is fitted with $k_{t}=a\left(x-x_{0}\right)^{-n}$, where $a=1.10, x_{0}=-0.35$ and $n=2.51$.

and the effect of the boundary condition at $x=0$ has disappeared. Therefore, the difference in viscosity between the inflow field and the actual simulation will have no influence at the position of the flame front, i.e. around $x=2.66$.

\subsection{Results of type I simulations (decay of incompressible isotropic turbulence)}

As a reference case we have computed the spatial decay of isotropic turbulence, which can be considered as a test of the numerical code. In figure 4, the decay of turbulent kinetic energy and its three components are shown as a function of the distance from the inflow boundary. The three components have about the same magnitude during the whole decay, which indicates that the turbulence indeed remains isotropic. By fitting a power law to the computational result, we find that the turbulent kinetic energy decays as $x^{-n}$ with $n=2.51$. This value is somewhat higher than the value given in, for example, George (1992), who mentions $n \simeq 2.1$.

From this decay, can estimate the turbulent dissipation $\epsilon$ with the help of the Taylor hypothesis as:

$$
\epsilon=-\frac{\mathrm{d} k}{\mathrm{~d} t}=-U_{\text {in }} \frac{\mathrm{d} k}{\mathrm{~d} x} .
$$

The Kolmogorov length scale $\eta$, scaled with the size of the domain is then obtained from

$$
\frac{\eta}{L_{y}}=\left(\frac{v^{\prime 3}}{\ell \epsilon} R e_{\ell}^{-3}\right)^{1 / 4} \frac{\ell}{L_{y}} .
$$

The $\epsilon$ and $\eta$ are both plotted in figure 5. For $x>4$, we find $\eta>0.01$. With the help of the value for $\eta$ estimated above, we can determine whether our DNS is fully resolved. The grid spacing in the $x$-directions is 0.0104 and in the $y$-and $z$-directions 0.0078 and these values are of the same order as the estimated Kolmogorov length scale. This means that our simulation cannot be regarded as fully resolved up to the Kolmogorov length. Nevertheless, we feel that the resolution is sufficient to describe the relevant turbulence dynamics in view of the remarks made by Moin \& Mahesh (1998). 


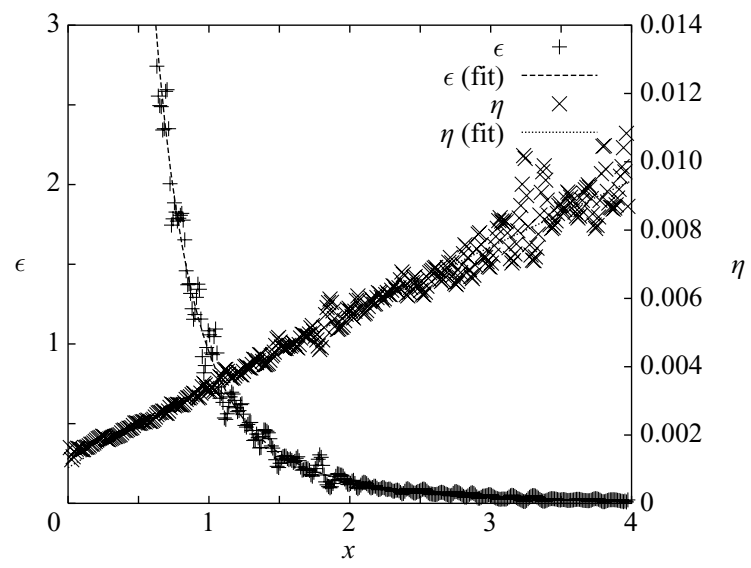

FigurE 5. The turbulent dissipation $\epsilon$ and the corresponding Kolmogorov length $\eta$ as a function of $x$. The curves are obtained from the expression of the decay of kinetic energy, determined in figure 4 , which leads to $\epsilon=2.75(x+0.35)^{-3.51}$.

$\begin{array}{ccccc}\begin{array}{c}\text { Heat release } \\ \text { parameter } \tau\end{array} & \begin{array}{c}\text { Number of } \\ \text { waves } n=L_{y} / \lambda\end{array} & \begin{array}{c}\text { Wave- } \\ \text { number } k\end{array} & \begin{array}{c}\text { Flame } \\ \text { thickness } \delta\end{array} & \text { Figure } \\ 1.0 & 2 & 4 \pi & 0.02 & 7,8 \text { and } 9 \\ 3.0 & 2 & 4 \pi & 0.02 & 7 \\ 5.0 & 2 & 4 \pi & 0.02 & 7 \\ 3.0 & 1 & 2 \pi & 0.02 & 8 \\ 3.0 & 3 & 6 \pi & 0.02 & 8 \\ 3.0 & 4 & 8 \pi & 0.02 & 8 \\ 1.0 & 2 & 4 \pi & 0.04 & 9 \\ 1.0 & 2 & 4 \pi & 0.1 & 9\end{array}$

TABLE 2. The different values of the parameters $\tau, k$ and $\delta$ used to study the growth of the disturbances by hydrodynamic instability of the flame front. The initial amplitude is always chosen as $A_{0}=0.001$, except for the case $n=1$, where $A_{0}=0.004$ is used.

\subsection{Results of type III simulation (flame front instability)}

Let us now consider the simulation of the hydrodynamic instability discussed in $\S 2.2$. The comparison of this simulation with the theory of $\S 2.2$ is a check whether compressibility effects are realistically represented by our model and whether these are correctly incorporated in our numerical code. A series of simulations is carried out in the two-dimensional geometry, described in $\S 5.1$. The initial value for the flow field is an undisturbed velocity $\boldsymbol{u}_{0}=\left(s_{f l}, 0,0\right)$. The flame front is put at $x_{0}=1.5$ and on this flame front we impose a small wavelike perturbation with a dimensionless wave length $\lambda$ and an amplitude $A_{0}$. This leads to a $G$-field equal to $G=x-x_{0}+A_{0} \sin (k y)$ where $k$ is the wavenumber $k=2 \pi / \lambda$. Note that $1 / \lambda=n$ is the number of waves in the domain. The initial amplitude is set equal to $A_{0}=0.001$, so that $k A_{0} \ll 1$ and $|\nabla G| \approx 1$. The position of the flame front is not stabilized, but $U_{i n}=s_{f l}$ is kept constant.

We have varied parameters $\tau, k$ and $\delta$ in order to study the influence of these parameters on the growth of the disturbances. In table 2, the parameters used in the various simulations are given. 


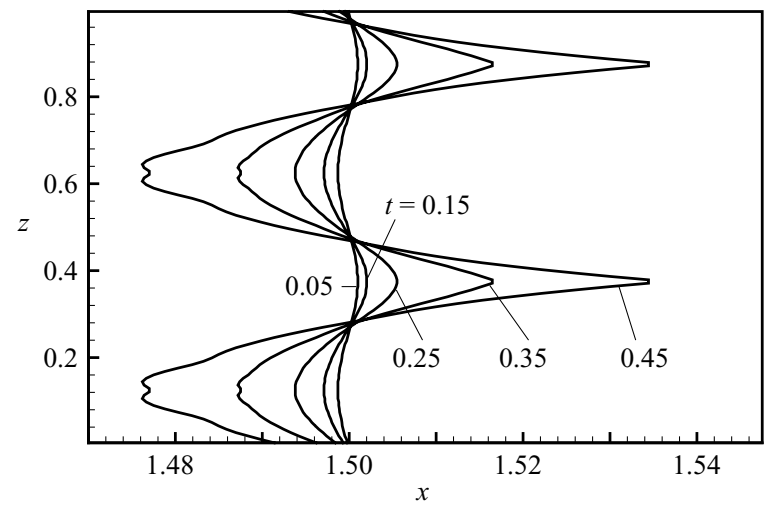

FIGURE 6. The evolution of the wave-like disturbance on a flat flame front with $n=2$ and $A_{0}=0.001$ at $t=0.05,0.15,0.25,0.35$ and 0.45 . The unburnt gas is on the left and the burnt gas on the right.

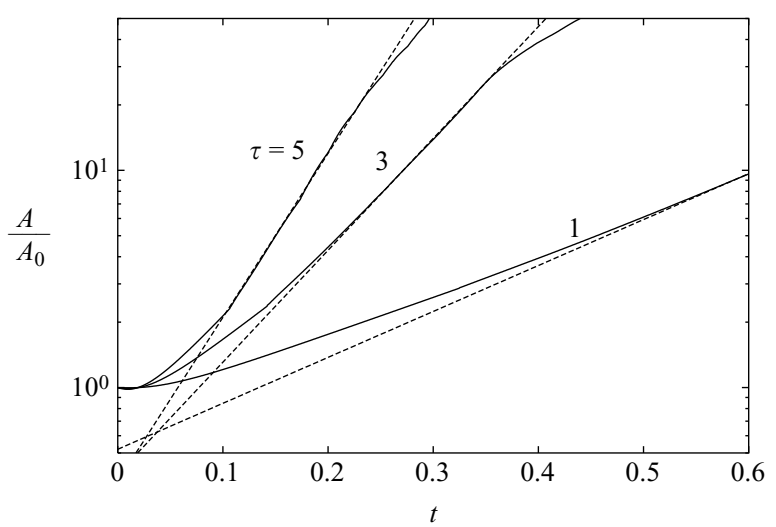

FIgURE 7. The normalized amplitude $A / A_{0}$ as a function of time for several values of the expansion ratio or heat release parameter, $\tau=1, \tau=3$ and 5. In all cases, $n=2, \delta=0.0$. The dashed line gives the theoretical growth rate. -, $A / A_{0}$ (computed);---, $A / A_{0} \sim \exp (\Omega t)$.

In figure 6, the evolution of the flame front is plotted for several times after $t=0$ for the case $\tau=3$. In the initial stages of the growth, the disturbance keeps its wavelike form, but at later times the shape starts to deviate from a wave owing to nonlinearity. The flame front develops sharp spikes, pointing in the direction of the burnt gas. This shape is similar to the flame-front pattern that we have shown in figure 2 where we have argued that this results from the flame propagating with respect to the unburnt gas in the direction normal to the flame front. Therefore, the results shown in figure 6 suggest that the instability will eventually be saturated by the flame front restoration process.

Between $t=0.05$ and $t=0.15$, the growth of the amplitude $A=\left(x_{f, \max }-x_{f, \min }\right) / 2$ is small, but thereafter the growth increases in time and becomes exponential as shown in figure 7 where we illustrate also the amplitude growth for other values of the expansion ratio or heat release parameter, i.e. $\tau=1$ and 5. After the velocity field has developed, figure 7 shows that the agreement between the growth rate found from the simulations and the theoretical growth rate is excellent. The results also show the expected increase in growth rate when $\tau$ becomes larger. At a later time, the growth rate obtained from 


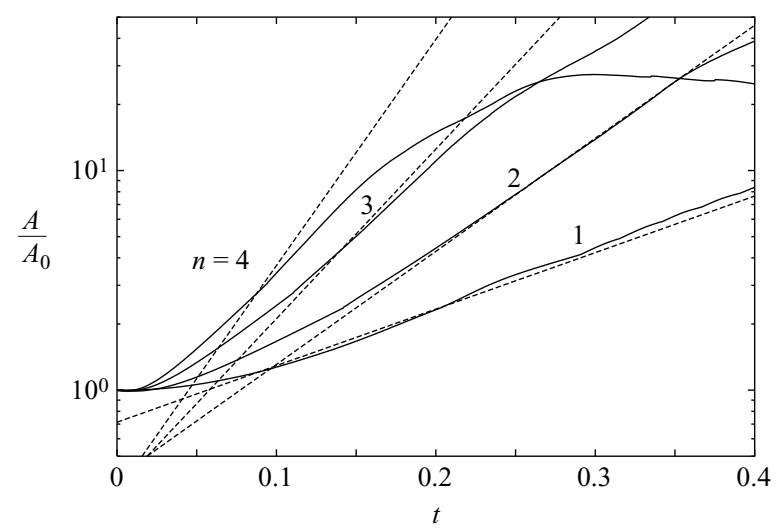

Figure 8. The normalized amplitude $A / A_{0}$ as a function of time for $n=1,2,3$ and 4 . For all cases, $\tau=3, \delta=0.02$. The dashed line indicates the theoretical growth rate. Key as figure 7 .

the simulations levels off and becomes smaller than the theoretical value. This is because nonlinear effects such as flame front restoration, mentioned above, set in.

Furthermore, we see in figure 6 that for $t \geqslant 0.25$, additional small disturbances appear on the flame front. These disturbances are probably the result of small discretization or numerical errors. Namely, it follows from (2.8) that the growth increases with $k$. Therefore, these errors, which have a large value of $k$ and which are initially very small, become visible in the final stages of the simulation. However, remember that in our case the Markstein length is taken equal to zero. When the Markstein length has a value unequal to zero, these small-scale perturbations can be removed by the diffusion process discussed in $\S 3.1$.

According to equation (2.8), the growth rate also depends on the wavelength of the disturbance and it increases for larger values of $n$ or $k=2 \pi n$. In figure 8 , the growth of the disturbance amplitude is plotted for several values of the wavenumber. For $n=1$ and $n=2$, the results show the expected increase with wavenumber and the agreement between the theory and the actual growth rate is very good as soon as the flow field has established itself. However, for shorter wavelengths, i.e. $n=3$ and $n=4$, the computed growth rate deviates from the theory. This is because in the theory one assumes that the flame is infinitely thin, but in our calculations this is not the case. The correctness of this explanation becomes clearer in figure 9, where the normalized amplitude is plotted as a function of time for one value of $n$ and $\tau$, but for several values of the flame thickness $\delta$. In all the previous graphs, the default value of $\delta=0.02$ has been used. When we use larger values such as $\delta=0.04$ and 0.1 , figure 9 shows that the growth rate decreases. Apparently, the growth rate is only close to the theoretical value $(2.8)$, if $\delta / \lambda \ll 1$. When either $\delta$ increases or $\lambda$ decreases, the actual growth rate becomes lower than the theoretical value, as we have also noticed in figure 8 .

Although the growth rate increases with increasing wavenumber, figure 8 shows that the final amplitude of disturbances with a short wavelength may become smaller than the final amplitude of longer waves. This might be problematic in numerical simulations which use periodic boundary conditions. In that case, the largest wavelength possible is a wavelength equal to the size of the numerical domain. Since a disturbance with this wavelength may continue to grow, it might start to dominate the solution, which eventually results in a solution, which is influenced by the size of the numerical domain. 


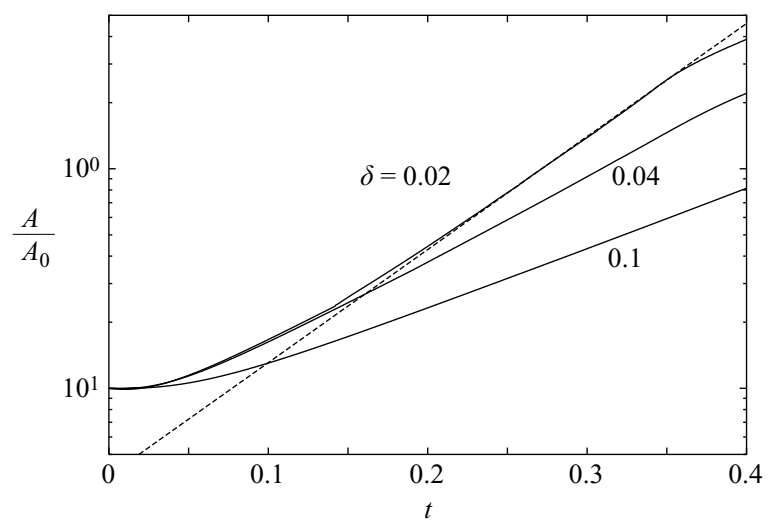

FIGURE 9. The normalized amplitude $A / A_{0}$ as a function of time for several values of the flame thickness $\delta=0.02,0.04$ and 0.1 . In all cases, $\tau=3$ and $n=2$. The dashed line indicates the theoretical growth rate. Key as figure 7.

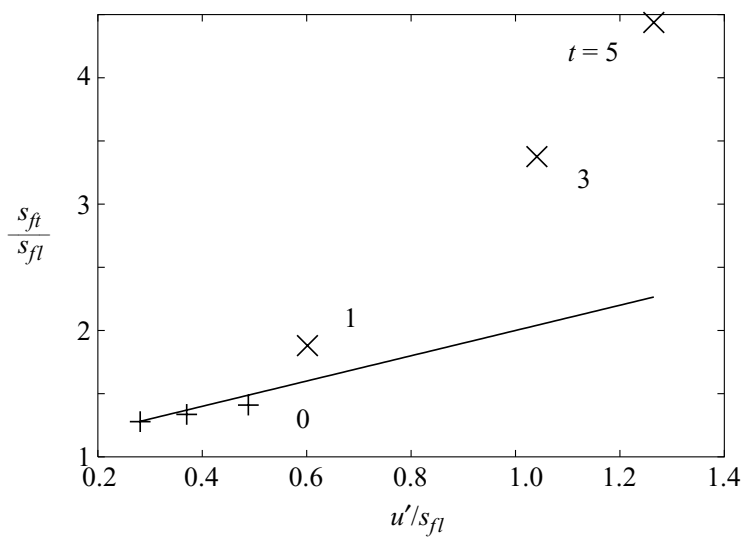

FIGURE 10. The turbulent flame speed $s_{f t}$ as a function of the turbulent intensity $u^{\prime}$, together with the expected profile, equation (2.6), with $C=1 .+$, results of simulation without expansion or $\tau=0 ; \times$, results of simulations with expansion.,$- s_{f t} / s_{f l}=1+C u$.

\subsection{Results of type IV simulations (turbulent premixed combustion)}

We now consider the DNS results, in which the full interaction between turbulence and the flame front is taken into account.

Let us first look at the influence of turbulence on the flame front, decoupled from the effect of volume expansion, i.e. without density fluctuations. To this end we have performed three simulations without expansion, i.e. $\tau=0$, and varying turbulence intensity at the inflow. The resulting turbulent flame speed $s_{f t}$ as a function of the turbulence intensity $u^{\prime}$ is plotted in figure 10 with the + symbols. Here, $u^{\prime}$ is defined as r.m.s. the value of the velocity fluctuations at the mean position of the flame front, $\overline{x_{f}}$, and is given by,

$$
u^{\prime}=\sqrt{\overline{u_{i}^{2}}}=\sqrt{2 \frac{\overline{k_{t}}}{\rho}},
$$

where the latter equality is valid only for this particular case for which $\tau=0$. 


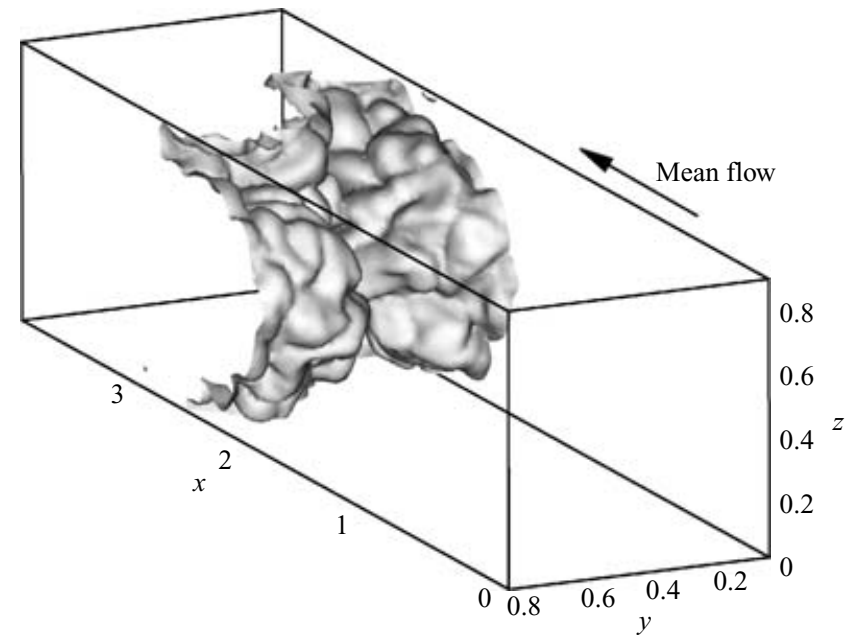

FigURE 11. An instantaneous picture of the flame front for $\tau=3$.

Figure 10 shows indeed an increase in flame speed with the level of turbulence as predicted by Damköhler (1940). The relation (2.6) with $C=1$ agrees reasonably well with data, although for the larger values of $u^{\prime}$ the computed turbulent flame speed seems to fall a little below the linear profile. This latter effect is probably due to the artificial thickness of the flame $\delta$, because for a strongly wrinkled flame, the increase of the flame surface is limited by $\delta$ and therefore the flame speed decreases.

Next we have carried out a series of simulations to consider the effect of gas expansion. An example of the instantaneous flame front for the case $\tau=3$ is shown in figure 11. The flame front is strongly deformed and we expect that this will lead to a strong increase of the turbulent flame speed.

The results for the simulations with gas expansion, i.e. $\tau \neq 0$ are also shown in figure 10 by the $\times$ symbols. The flame speed has clearly increased. If we assume that this increase results solely from the turbulent distortion of the flame front, the data must fall along the linear line (2.6) discussed above. We see, however, that this is not the case. So it appears that $s_{f t}$ also depends directly on the heat release parameter $\tau$.

Let us assume that (2.6) is still a valid approximation for the influence of turbulence on the flame speed in the range of $u^{\prime} / s_{f l}$ that are found for the cases $\tau \neq 0$. The direct effect on the flame speed by the gas expansion can then be described by plotting the constant $C$ defined as $C=\left(s_{f t}-s_{f l}\right) / u^{\prime}$ as a function of $\tau$. This is shown in figure 12 where we see that increase of the turbulent flame speed grows when the expansion rate rate becomes larger. The rate of growth of $C$ seems to level off at the higher expansion rates. These results are consistent with the results of Wenzel \& Peters (2000), who also observed an additional increase in the turbulent flame speed for higher expansion rates. Their conclusion, that the effect of gas expansion is important only for low turbulence intensities could not be validated here, since $u^{\prime}$ is low in all our simulations.

The explanation of this dependence of the turbulent flame speed on the gas expansion must be sought in the effect of the hydrodynamic instability. We have seen in $\S 5.3$ that disturbances on the flame front will grow, causing an increase in the area of the flame front and therefore an increase in the turbulent flame speed. The results of $\S 5.3$ also indicate that the hydrodynamic instability of the flame front becomes stronger at higher expansion rates in agreement with the behaviour of our data in figures 10 and 12. 


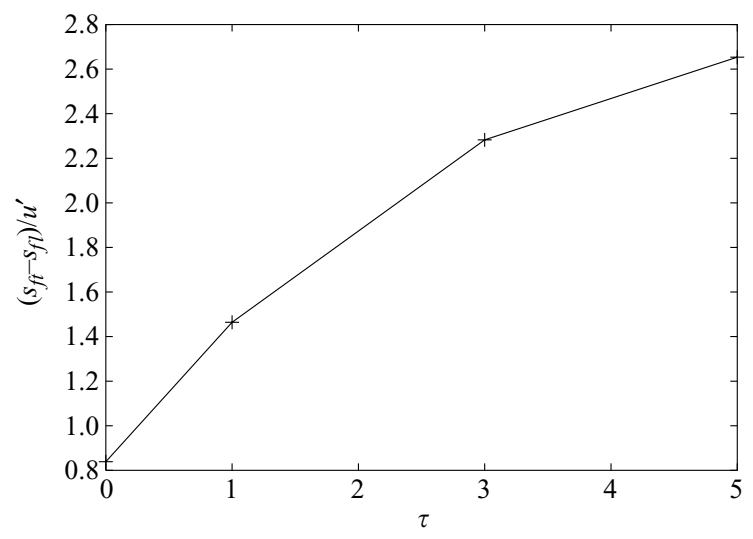

FIGURE 12. The increase of $C=\left(s_{f t}-s_{f l}\right) / u^{\prime}$ as a function of the heat release parameter $\tau$.

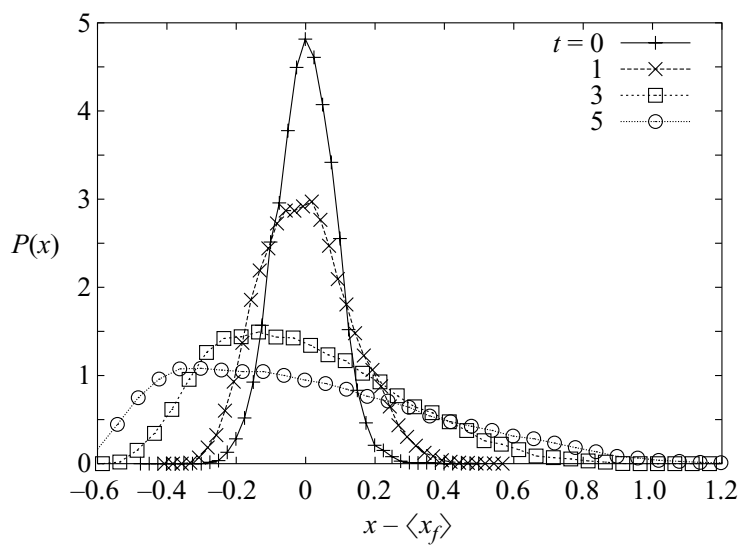

FIGURE 13. The probability density of finding the instantaneous flame front at a position $x$ with respect to the mean position $\overline{x_{f}}$ for several values of the heat release parameter $\tau$.

\subsection{The position of the flame front $x_{f}$}

As can be seen in figure 11, the flame front is strongly deformed. As a result, the $x$ position of the instantaneous flame front, $x_{f}$, depends on the $y$-and $z$-coordinate and the time. In figure 13, we have plotted the probability density of finding the instantaneous flame front at a certain position $x$. At $\tau=0$, i.e. without expansion, the distribution is nearly symmetric with respect to $x_{f}=\overline{x_{f}}$. When $\tau \neq 0$, the distribution broadens as a function of $\tau$ and at the same time becomes positively skewed. This means that the disturbances on the flame front grow with larger values of $\tau$. This is in agreement with the fact that the growth rate of hydrodynamic instabilities becomes larger when $\tau$ increases. The positive skewness, i.e. large excursions of the flame front to the burnt gas side, is in agreement with results of Wirth, Keller \& Peters (1993) and experiments of Plessing et al. (2000); it is due to the flame developing rounded edges toward the unburnt gas and sharp spikes toward the burnt gas (figure 6).

\subsection{Change of turbulence characteristics across the flame front}

Let us now consider how the turbulence changes when the flow passes the flame front. First, we consider the turbulent kinetic energy. In figure 14, the mean turbulent 

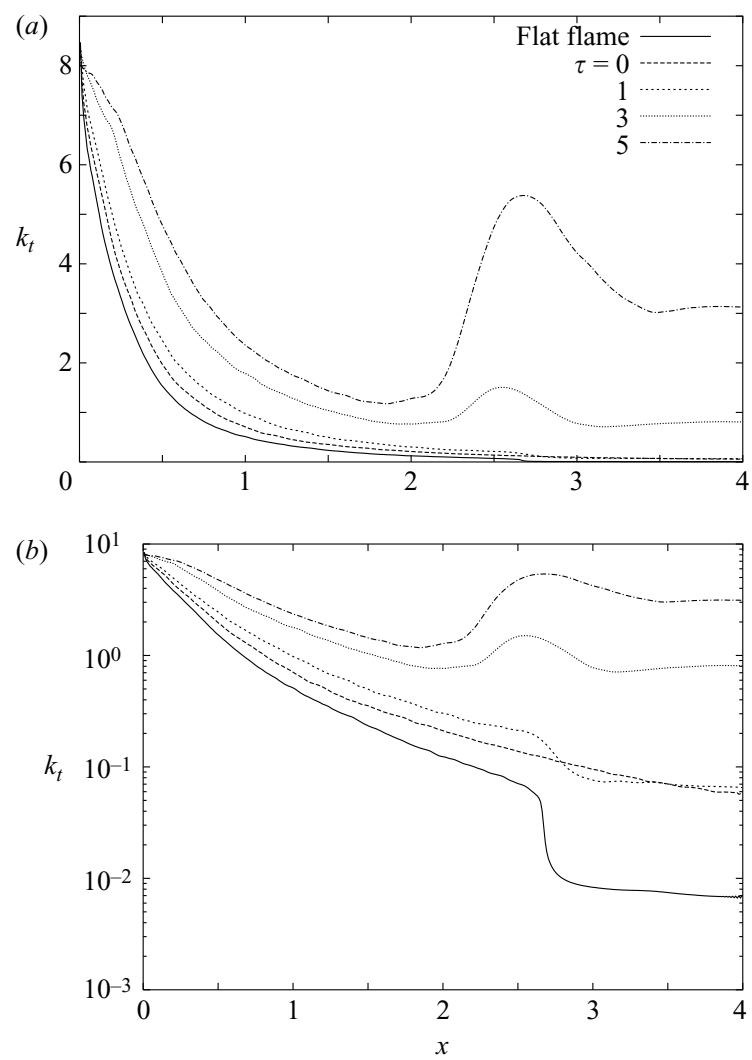

Figure 14. The turbulent kinetic energy $\bar{k}_{t}$ as a function of $x$ for various values of $\tau$. The initial condition at $x=0$ is the same for all cases. In addition, we show the results for the case without combustion and for the simulation with the flat flame front. The flat flame shows a strong suppression of turbulence, while the turbulent kinetic energy is enhanced in case of a wrinkled flame at large values of $\tau$. (a) Normal scale; $(b) \log$ scale.

kinetic energy per unit volume $\bar{k}_{t}$ defined according to (3.22) is plotted as a function of $x$ for several expansion rates. The initial condition for the turbulence intensity at the inflow is chosen to be the same for all cases. For the case $\tau=0$, there is no gas expansion and the development of the turbulence will not be affected in this case, i.e. the decay of turbulence will be similar to that found in figures 4 for the incompressible case. For the case $\tau=1$, we see an decrease of the turbulent kinetic energy per unit volume, when the turbulence passes the flame front. However, for higher expansion rates, $\tau=3$ and $\tau=5$, this turns into an increase in the turbulent kinetic energy.

In order to throw more light on this change of behaviour as a function of $\tau$, we also performed a simulation of turbulence passing a flat flame, which is denoted as type II in table 1. This means that the gas expands at a fixed flame position and the shape of the flame front is thus not influenced by the turbulence or instability processes. For the expansion rate in the case of the flat flame, we take $\tau=3$. We see in figure 14 that the kinetic energy strongly decreases when it passes the flat flame front. The conclusion is that the decrease of the kinetic energy across the flame front must be connected to the gas expansion and the increase of kinetic energy must be related to deformation of the flame front which occurs in particular at the higher expansion rates. We shall return to this when we discuss the kinetic energy budget in $\S 5.7$. 


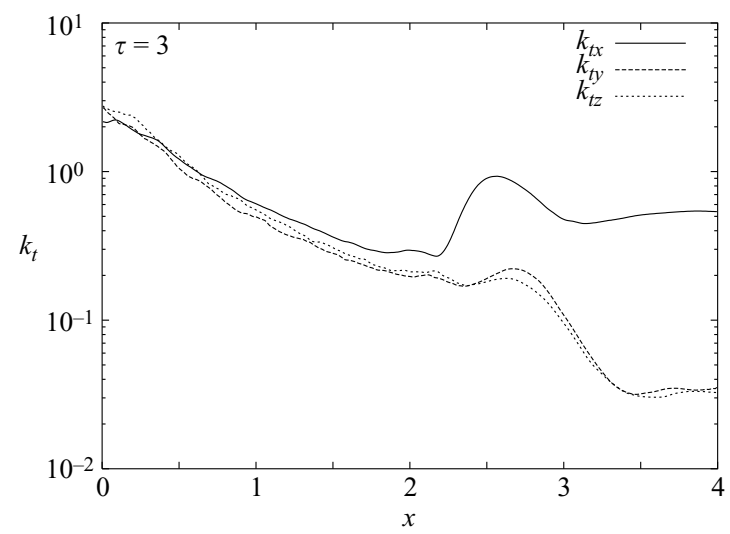

FIGURE 15. The different components of the turbulent kinetic energy as a function of $x$ for $\tau=3 . k_{t x}=\frac{1}{2} \overline{\rho u_{1}^{\prime} u_{1}^{\prime}}, k_{t y}=\frac{1}{2} \overline{\rho u_{2}^{\prime} u_{2}^{\prime}}$ and $k_{t z}=\frac{1}{2} \overline{\rho u_{3}^{\prime} u_{3}^{\prime}}$.

In figure 15, we have plotted the different components of the turbulent energy for the case $\tau=3$. Before the flame front, the three components are almost equal which implies isotropic conditions. Behind the flame front, the $x$-component of the turbulent energy strongly increases while the other contributions continue to decay. As a result, the turbulence behind the flame front becomes strongly anisotropic and it seems that there is no tendency to return to isotropy further downstream.

The increase of the turbulent kinetic energy is thus solely due to the growth of the velocity fluctuations in the $x$-direction. This points again in the direction of the hydrodynamic instability as being the cause for this growth because we have seen in $\S 5.3$ that the hydrodynamic instability influences primarily the $x$-component of the velocity. We shall come back to this issue in the next section when we discuss the kinetic energy budget.

\subsection{Turbulent kinetic energy budget}

To investigate the background behind the behaviour of the turbulent kinetic energy that we observed in the previous section near the flame front, in more detail, we consider in this section the different terms in the budget for the turbulent kinetic energy as expressed in (3.27) (see also Rutland \& Cant 1994). These terms are plotted in figure 16 for various values of the expansion parameter $\tau$. The diffusive transport term $D_{k}$ has been neglected because this term is small since there are no regions of high shear in the mean flow field.

In the case of $\tau=0$, (figure 16a), the main balance is between the transport of turbulent kinetic energy by the mean flow, $\mathrm{D} k / \mathrm{D} t$, and viscous dissipation, $\epsilon_{k}$. The fluctuations in $\mathrm{D} k / \mathrm{D} t$ are due to the statistical error in $\overline{k_{t}}$, which is amplified by $\mathrm{D} k / \mathrm{D} t$ being computed by taking a spatial derivative of $\overline{k_{t}}$. The total budget term is not exactly zero in figure 16 . This is caused by the fluctuations in the $\mathrm{D} k / \mathrm{D} t$-term and by the stabilization mechanism keeping the flame front at a fixed position causing a slowly varying mean flow with a long time scale. As a result the statistics converge slowly.

In case of $\tau=1$ (figure $16 b$ ), the $\mathrm{D} k / \mathrm{D} t$ and the $\epsilon_{k}$-term remain important, but contributions of the expansion term $E_{k}$ and the pressure term $\Pi_{k}$ become non-negligible. The $\Pi_{k}$ has a positive and negative contribution which seems to cancel one another. The $E_{k}$ is everywhere negative which we have also observed in relation to (3.27) for this case where $\partial \bar{u}_{i} / \partial x_{i}>0$. Apparently, the expansion term $E_{k}$ accounts for the 

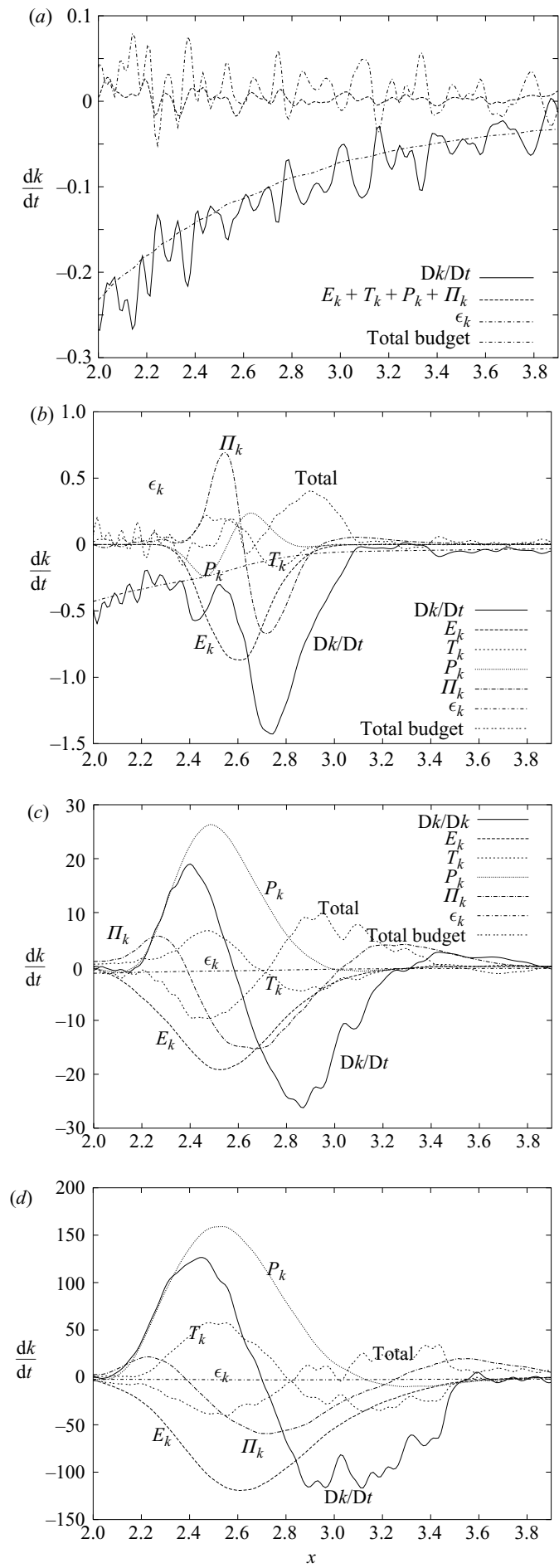

FIGURE 16. Kinetic energy budget for several expansion rates. $(a) \tau=0 ;(b) 1 ;(c) 3 ;(d) 5$. 


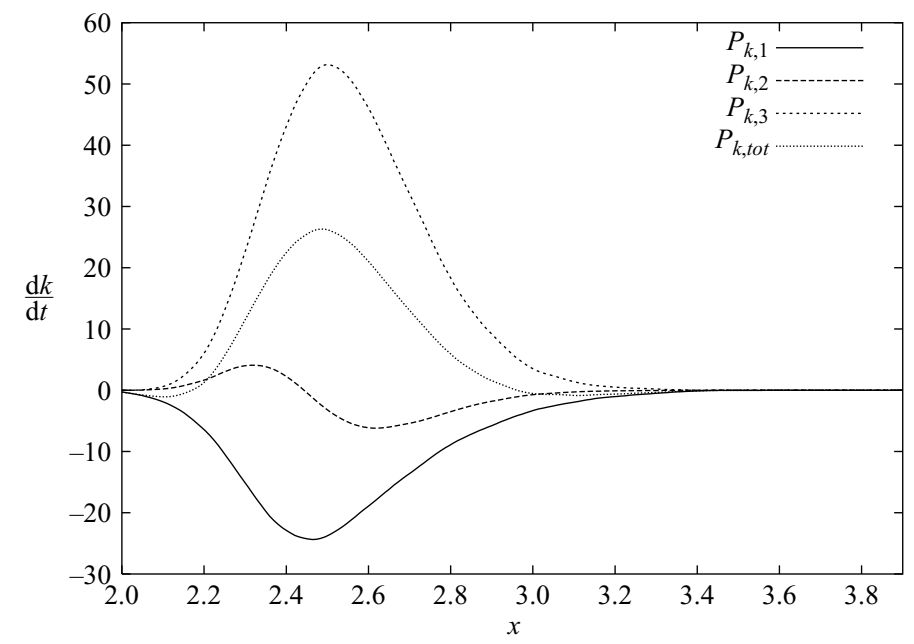

FIgURE 17. The different term in the production term $P_{k}$ of the turbulent kinetic energy as a function of $x$ for $\tau=3 . P_{k, 1}=-\bar{\rho} \overline{u_{i}^{\prime} u_{j}^{\prime}}\left(\partial \bar{u}_{i} / \partial x_{j}\right), P_{k, 2}=-\overline{\rho^{\prime} u_{i}^{\prime} u_{j}^{\prime}}\left(\partial \bar{u}_{i} / \partial x_{j}\right) P_{k, 3}=-\overline{\rho^{\prime} u_{i}^{\prime}} \overline{u_{j}}\left(\partial \bar{u}_{i} /\right.$ $\left.\partial x_{j}\right)$ and $P_{k, t o t}$ is the sum of these terms.

decrease of turbulent kinetic energy that we observe in figure 14 which is consistent with our observation based on the flat-flame results shown in this figure. A decrease of kinetic energy has also been observed in previous studies, e.g. by Lindstedt \& Sakthitharan (1998). In that case, the decrease of turbulent kinetic energy across a flame front is attributed to an increased viscosity, which accompanies the temperature rise, due to combustion. In our case, the viscosity is taken as constant and thus cannot play a role. Therefore, our results indicate that the decrease of energy observed may also result from the gas expansion.

At the larger expansion rates $\tau=3$ and 5 (figure $16 c, d$ ), the picture changes. Both terms $E_{k}$ and $\Pi_{k}$ remain important, but the production term $P_{k}$ grows and becomes the dominant term. In the region $x=2.0-2.2$, the terms $E_{k}$ and $\Pi_{k}$ are in balance and the $\mathrm{D} k / \mathrm{D} t$-term almost coincides with the production term $P_{k}$. This explains the increase in the kinetic energy that we have observed in figure 14 for $\tau=3$ and 5 .

To explain the background of this increase in the production $P_{k}$ we consider the three terms that contribute to $P_{k}$, separately. These are

$$
\left.\begin{array}{c}
P_{k, 1}=-\bar{\rho} \overline{u_{i}^{\prime} u_{j}^{\prime}} \frac{\partial \bar{u}_{i}}{\partial x_{j}}, \\
P_{k, 2}=-\overline{\rho^{\prime} u_{i}^{\prime} u_{j}^{\prime}} \frac{\partial \bar{u}_{i}}{\partial x_{j}}, \\
P_{k, 3}=-\overline{\rho^{\prime} u_{i}^{\prime} \bar{u}_{j}} \frac{\partial \bar{u}_{i}}{\partial x_{j}} .
\end{array}\right\}
$$

For the case $\tau=3$ we have plotted these contributions to $P_{k}$ in figure 17. We find that the dominant contributions are both $P_{k, 1}$ and $P_{k, 3}$ with $P_{k, 2}$ being much weaker. The $P_{k, 1}$ is negative, but the $P_{k, 3}$ is strongly positive and is therefore responsible for the positive total production $P_{k}$.

Given the definition of $P_{k, 3}$ in (5.9) and the fact that $\bar{u}_{i}=\left(\bar{u}_{1}, 0,0\right)$ with $\bar{u}_{1}>0$ and that $\partial \bar{u}_{1} / \partial x_{1}$ is also $>0$ as a result of the expansion, we find that a positive value of $P_{k, 3}$ can only result from a negative density transport term $\overline{\rho^{\prime} u_{i}^{\prime}}$. In $\S 2.2$, we found 


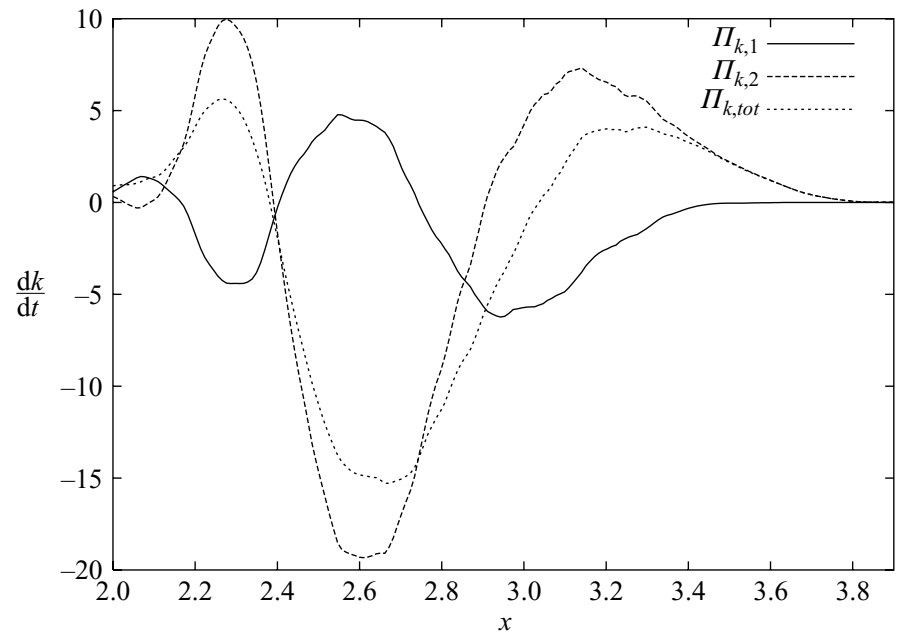

FigurE 18. The different term in the production term $\Pi_{k}$ of the turbulent kinetic energy as a function of $x$ for $\tau=3 . \Pi_{k, 1}=\overline{p^{\prime}\left(\partial u_{i}^{\prime} / \partial x_{i}\right)}, \Pi_{k, 2}=-\left(\partial \overline{p^{\prime} u_{i}^{\prime}} / \partial x_{i}\right)$ and $\Pi_{k, t o t}$ is the sum of these terms.

that such a negative density transport is consistent with the deformation of the flame front. Therefore we conclude that the increase of kinetic energy that we have observed at the high expansion rates is the result of the deformation of the flame front related to the Darrieus-Landau instability dicussed in $§ 2.2$ (see also Boughanem \& Trouvé 1998). This deformation occurs mainly in the streamwise direction which suggests that only the $u_{1}^{\prime}$ is influenced. This is consistent with the results shown in figure 15 .

Finally, we consider the pressure contribution $\Pi_{k}$ to the kinetic energy budget (3.27) in more detail. This term can be divided in two contributions

$$
\Pi_{k, 1}=\overline{p^{\prime} \frac{\partial u_{i}^{\prime}}{\partial x_{i}}}, \quad \Pi_{k, 2}=-\frac{\partial \overline{p^{\prime} u_{i}^{\prime}}}{\partial x_{i}},
$$

where $\Pi_{k, 1}$ is called the pressure dilatation and $\Pi_{k, 2}$ the pressure transport. These two contributions to $\Pi_{k}$ are shown in figure 18 for $\tau=3$. The $\Pi_{k}$ seems to be dominated by the pressure transport term. The pressure-dilation term which behaves with an opposite phase to the pressure transport term, has a maximum value around $x=2.6$, a value which appears to be somewhat larger than the position where $P_{k, 3}$ reaches its maximum. This suggest that this positive contribution of the pressure dilation term to the kinetic energy budget may depend on a process other than the flame-front deformation which, as we have seen above, dominates the $P_{k, 3}$. We shall come back to this in the next section.

\subsection{Enstrophy budget}

Another view on the dynamics of the turbulence in the neighbourhood of the flame front can be obtained by considering the enstrophy. In figure 19, we have plotted the enstrophy as a function of $x$ for the same cases as shown in figure 14 for the turbulent kinetic energy. The behaviour of the enstrophy follows closely that of the kinetic energy. In the case of $\tau=0$, the enstrophy shows a continuous decay along the $x$-axis. In the case of $\tau=1$, enstrophy is destroyed at the flame front and in the case of $\tau=3$ and $\tau=5$, enstrophy is generated. Also, the simulation of the flat 

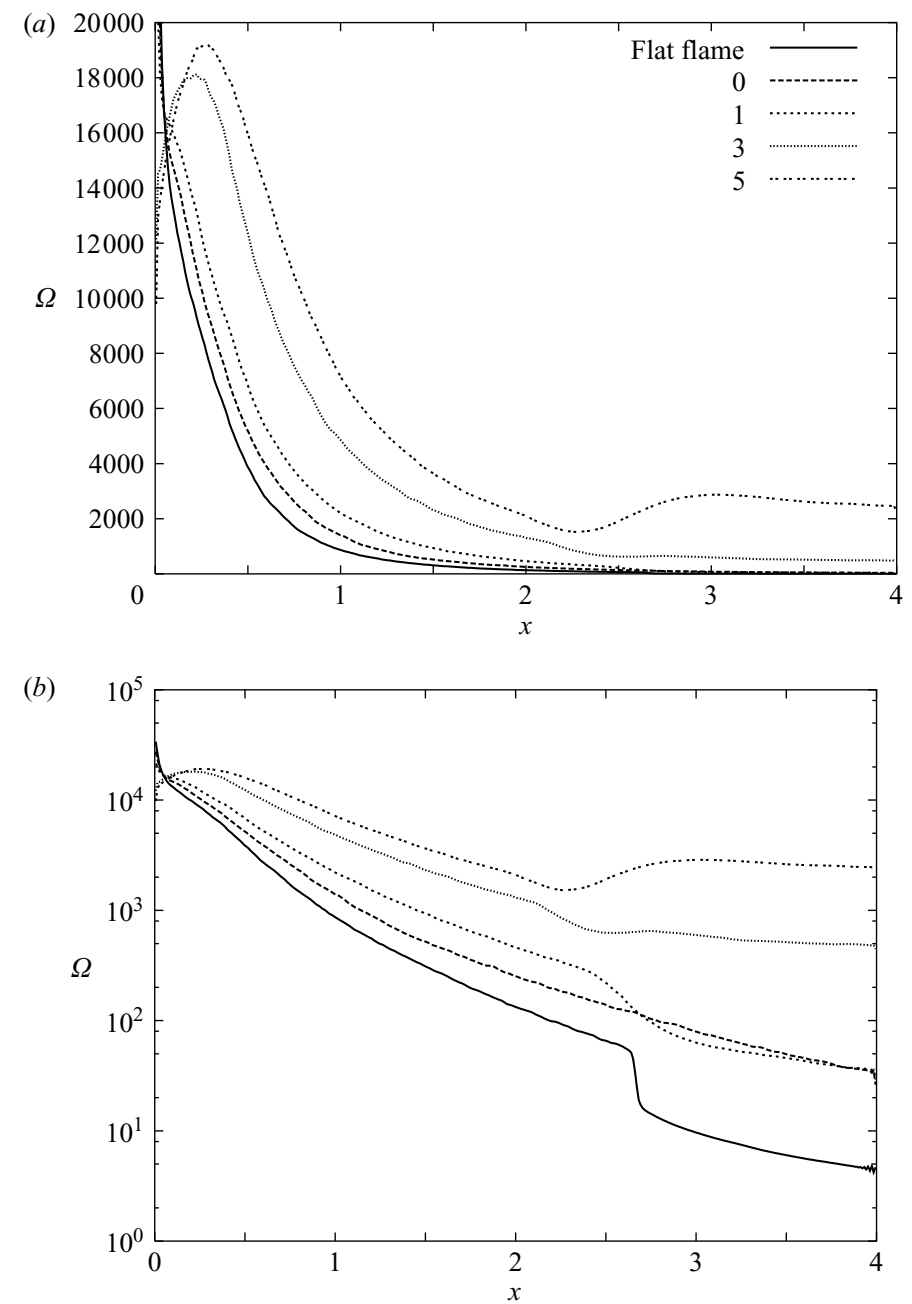

FIGURE 19. The enstrophy $\Omega$ as a function of $x$ for several expansion rates. (a) Normal scale; (b) log scale.

flame shows the same behaviour as for the kinetic energy, i.e. a strong decrease of the enstrophy at the flame front.

In figure 20, we have plotted the contribution of the different vorticity components to the enstrophy for $\tau=3$. This figure shows that the initially isotropic distribution of the enstrophy over its components becomes anisotropic when it crosses the flame front, i.e. the $z$ - and $y$-components are amplified while the $x$-component decreases further.

Let us interpret the behaviour of the enstrophy in terms of its budget (3.31). The terms in this budget are shown in figure 21 as a function of $x$, for several values of $\tau$.

In the case of $\tau=0$, there is a balance between production by vortex stretching $S_{\omega}$ and the viscous dissipation of enstrophy $\epsilon_{\omega}$ with a minor contribution by the mean transport term. The total budget is not zero in any of the graphs of figure 21 because $\epsilon_{\omega}$ is underestimated. Namely, $\epsilon_{\omega}$ is obtained from a spatial derivative of the vorticity fluctuations, which in their turn are obtained from a spatial derivative of the velocity field. Because we have used a staggered grid in our computations, certain 


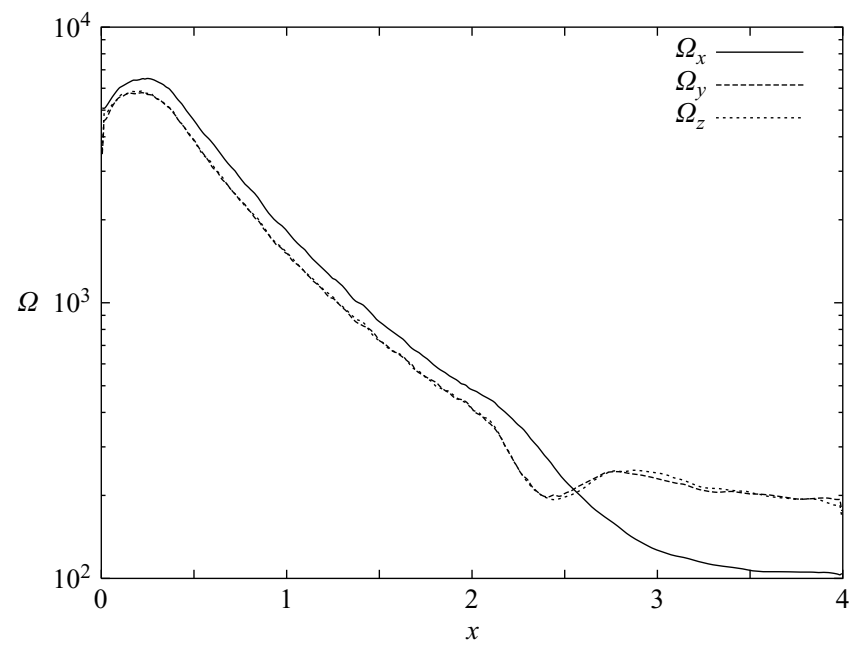

FIGURE 20. The contributions of the different vorticity components to the total enstrophy as a function of $x$ for $\tau=3 . \Omega_{x}=\frac{1}{2} \overline{\omega_{1}^{\prime} \omega_{1}^{\prime}}, \Omega_{y}=\frac{1}{2} \overline{\omega_{2}^{\prime} \omega_{2}^{\prime}}$ and $\Omega_{z}=\frac{1}{2} \overline{\omega_{3}^{\prime} \omega_{3}^{\prime}}$.

terms can only be obtained when the velocity field is interpolated. This interpolation is consistent with the discretization scheme used in the equations. Nevertheless, the interpolation smooths the velocity field and introduces an error which causes the higher derivatives, such as the enstrophy, to be underestimated.

In the case of $\tau=1$, the term $E_{\omega}$ becomes more important. This term accounts for the decrease of enstrophy density, owing to the expansion in the velocity field. This is the same effect as we saw in the energy budget. In the case of the higher expansion rates, i.e. $\tau=3$ and $\tau=5$, this term remains important and grows, but the baroclinic production term $\Pi_{\omega}$ grows even faster, resulting in the production of enstrophy at a position near the flame front.

By comparing figures 18 and 21, we observe that the maximum of $\Pi_{\omega}$ appears at the position where the pressure dilation term $\Pi_{k, 2}$ has its maximum. So these two terms seem to describe the same process, i.e. baroclinic production. However, we have seen also in the previous section that the pressure dilation term does not have much influence on the growth of the kinetic energy which is instead dominated by the production term $P_{k}$ or rather by the flame-front deformation. Therefore, we must conclude that the mechanism for the growth of the kinetic energy and the enstrophy are quite different, despite the fact that their behaviour as a function of $x$ is similar, as follows from comparing figures 14 and 19.

We have found that main production mechanism of enstrophy appears to be the baroclinic vorticity production $\Pi_{\omega}$. For a more detailed view of this process, we show figure 22(a) an instantaneous plot of lines of constant pressure, together with the position of the flame front. The isobars are nearly straight and do not follow the deformation of the flame front. Because the density gradient is perpendicular to the local flame front, the pressure and density gradient are clearly not aligned. This results in baroclinic vorticity production figure 22(b). Depending of the orientation of the flame, either positive or negative $\omega_{y}$ vorticity is produced and both contribute to the enstrophy. In the case of high expansion rates, this production of vorticity becomes important and influences the structure of the flow behind the flame front. In figure 23, we plot the modulus of the vorticity in an instantaneous $(x, z)$-slice, which also shows 

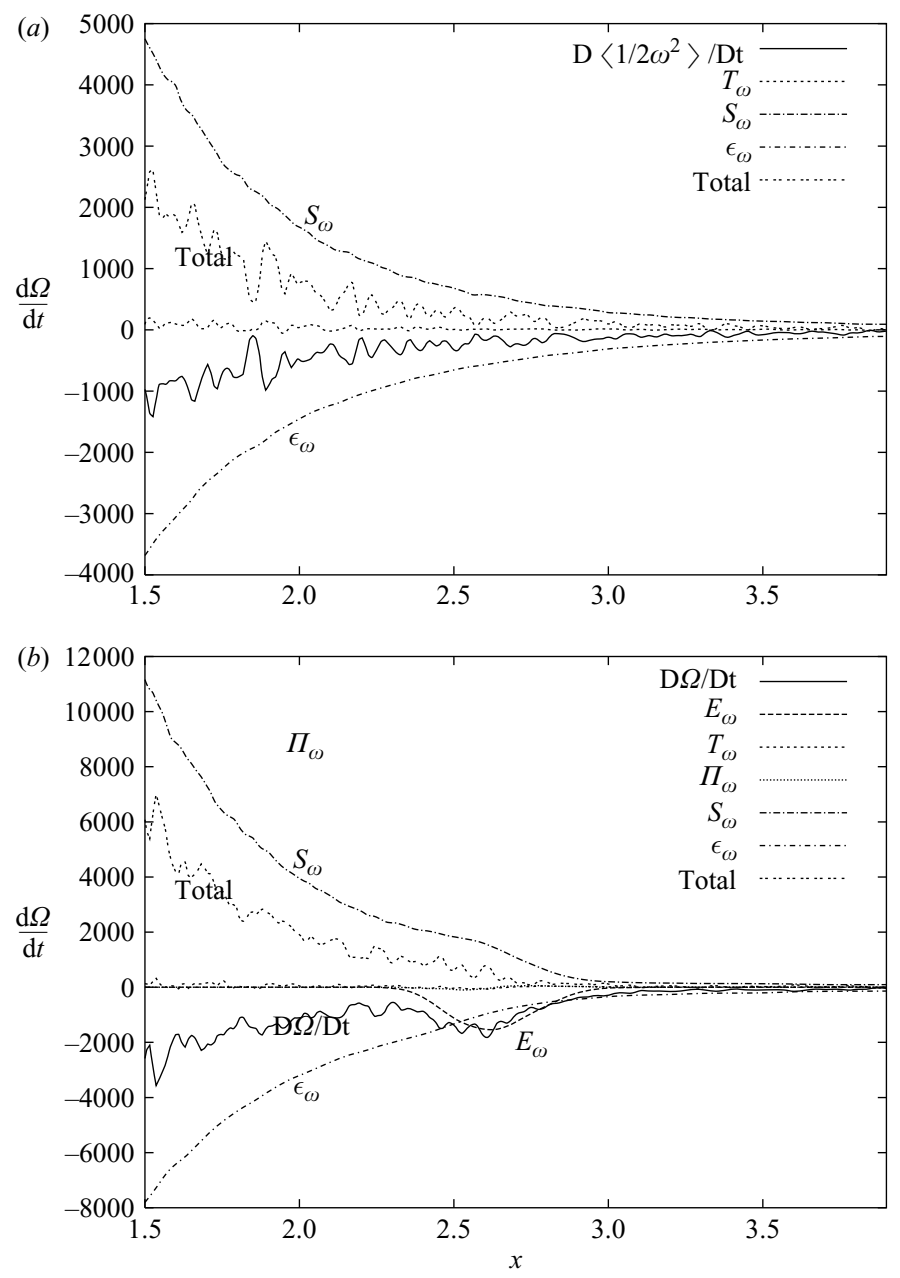

FIGURE 21 $(a, b)$. For caption see facing page.

that the vorticity in the flow behind the flame front has a different structure from the vorticity in front of the flame front.

The role of baroclinic vorticity production in the interaction between turbulence and a flame front is consistent with the investigations of Rutland \& Ferziger (1991); Mueller et al. (1998) and Louch \& Bray (1998), who studied the interaction between vortices and a premixed flame. They found that this results in long regions of vorticity in the streamwise direction, which can also be found in our simulation (figure 23).

\subsection{Favre averaging}

In $\S 3.5$, we mentioned that Favre averaging is often used in flows with a varying density. Since all the data required to obtain the Favre-averaged turbulent kinetic energy (3.34), are available from our DNS data, we can compare the kinetic energy defined in (3.22) with the Favre-average turbulent kinetic energy. Both are plotted in figure 24 as a function of $x$. The graphs in this figure have the same shape, but the peak of the Favre-averaged energy is about 20\% lower, which is consistent with (3.35). 

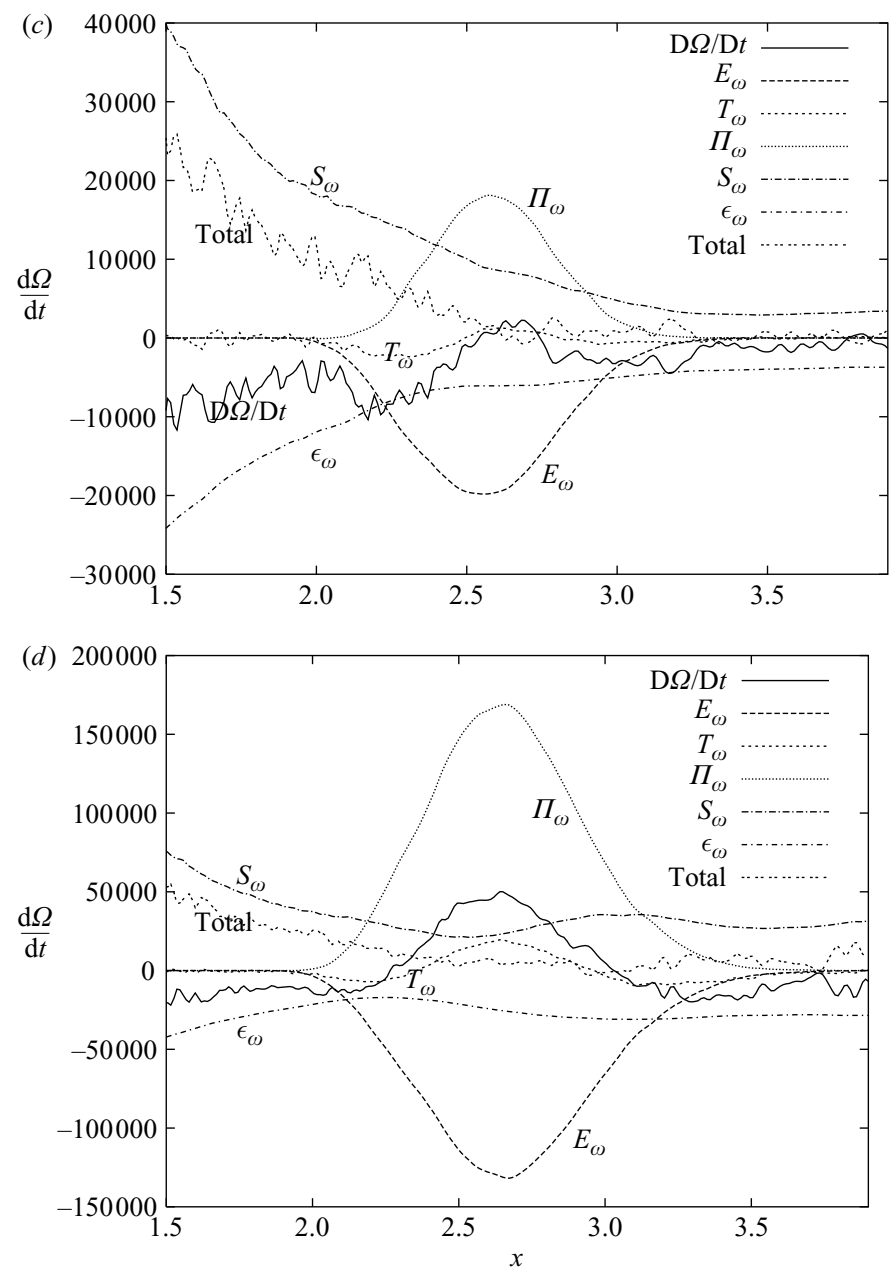

FIGURE 21. Enstrophy budget for several expansion rates. (a) $\tau=0 ;(b) 1 ;(c) 3 ;(d) 5$.

Another view on Favre averaging may be obtained by considering the budget of the Favre averaged kinetic energy $\widetilde{k}_{t}$ defined by (3.34) which reads (Lele 1994)

$$
\frac{\partial \widetilde{k}_{t}}{\partial t}+\frac{\partial \widetilde{u}_{j} \widetilde{k}_{t}}{\partial x_{j}}=-\frac{\partial \widetilde{k_{t}^{\prime \prime} u_{j}^{\prime \prime}}}{\partial x_{j}}-\bar{\rho} \widetilde{u_{i}^{\prime \prime} u_{j}^{\prime \prime}} \frac{\partial \widetilde{u}_{i}}{\partial x_{j}}+\overline{u_{i}^{\prime \prime} \frac{\partial p^{\prime}}{\partial x_{i}}}-\overline{u_{i}^{\prime \prime} \frac{\partial \tau_{i j}^{\prime}}{\partial x_{j}}}-\overline{u_{i}^{\prime \prime}} \frac{\partial \bar{p}}{\partial x_{i}}+\overline{u_{i}^{\prime \prime}} \frac{\partial \overline{\tau_{i j}}}{\partial x_{j}}
$$

with

$$
k_{t}^{\prime \prime}=\frac{1}{2} \rho u_{i}^{\prime \prime 2} .
$$

The first four terms on the right-hand side of this equation are the turbulent transport, the shear production, the pressure and the viscous terms, respectively. These terms appear also in the budget of kinetic energy for an incompressible flow. The remaining two terms, which have no equivalent in the incompressible energy budget, are a result of the Favre-averaging procedure or in particular of the fact that $\overline{u_{i}^{\prime \prime}} \neq 0$. Let us 

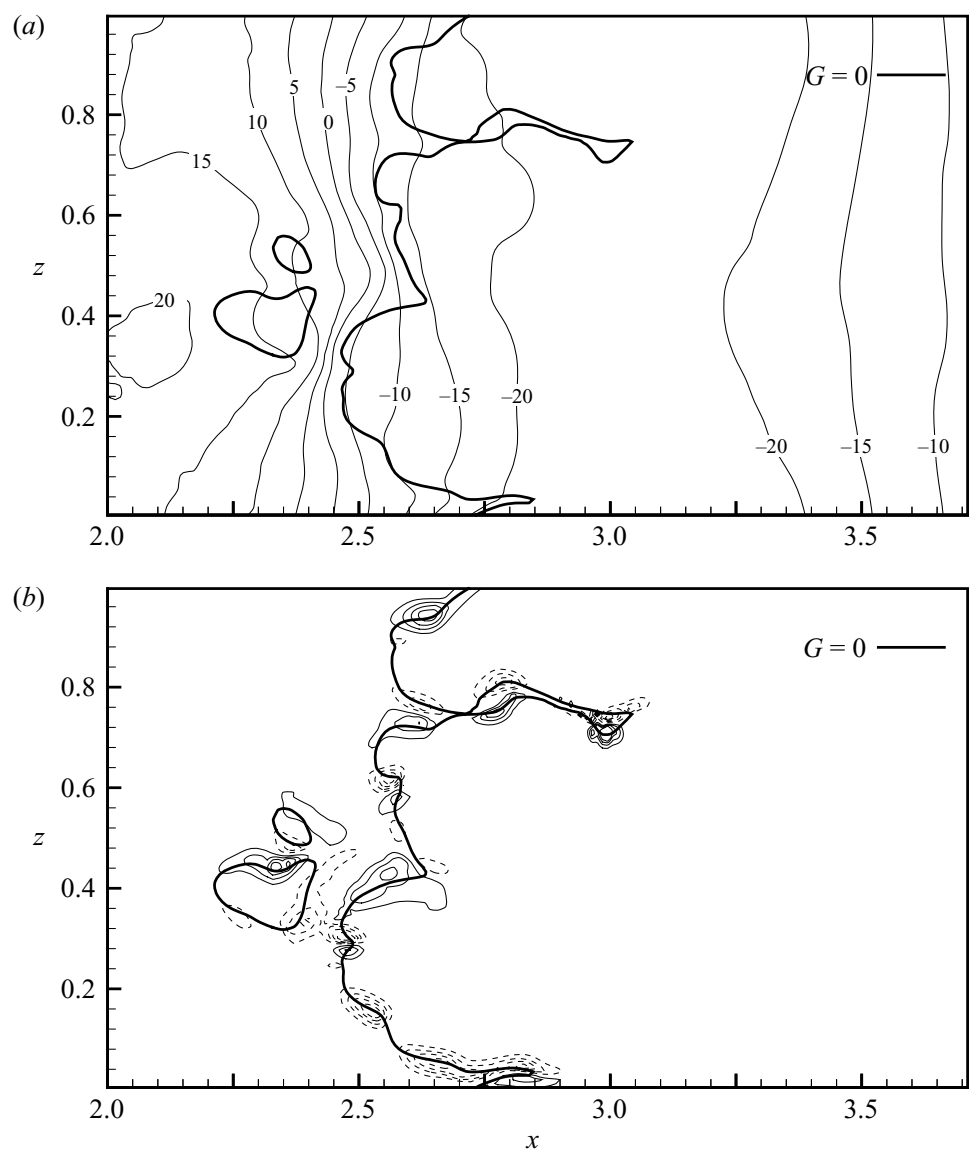

FiguRE 22. (a) Lines of constant pressure. (b) Regions of baroclinic vorticity production. The continuous lines indicate regions of positive $\omega_{y}$ production. The dashed lines indicate regions of negative $\omega_{y}$ production. In both plots the thick solid line indicates the position of the flame front in an instantaneous $x z$-slice at $\tau=3$.

consider these two terms in more detail. With help of the definition

$$
\overline{\rho^{\prime} u_{i}^{\prime}}=-\bar{\rho} \overline{u_{i}^{\prime \prime}}
$$

and the momentum equation (3.33), we can write these terms as

$$
-\overline{u_{i}^{\prime \prime}} \frac{\partial \bar{p}}{\partial x_{i}}+\overline{u_{i}^{\prime \prime}} \frac{\partial \overline{\tau_{i j}}}{\partial x_{j}}=-\overline{\rho^{\prime} u_{i}^{\prime \prime}} \frac{\partial \widetilde{u_{i}}}{\partial t}-\overline{\rho^{\prime} u_{i}^{\prime \prime}} \tilde{u_{j}} \frac{\partial \widetilde{u_{i}}}{\partial x_{j}}+\overline{u_{i}^{\prime \prime}} \frac{\partial \bar{\rho} \widetilde{u_{i}^{\prime \prime} u_{j}^{\prime \prime}}}{\partial x_{j}} .
$$

This equation shows that, besides a contribution due to a variation of the Reynolds stress, the last two terms in (5.11) are also responsible for the production term $P_{k, 3}$ which we have already encountered as part of the production term of the kinetic energy budget (3.27). We have seen that $P_{k, 3}$ describes the process of flame-front deformation, which plays an important role in the dynamics of turbulence near the flame front. This means that the last two terms on the right-hand side of (5.11) cannot be neglected as is also mentioned by Bray, Champion \& Libby (2000). Unless a model for these terms is adopted, the use of Favre averaging will not give a realistic representation of the flame front dynamics for the case studied here. 

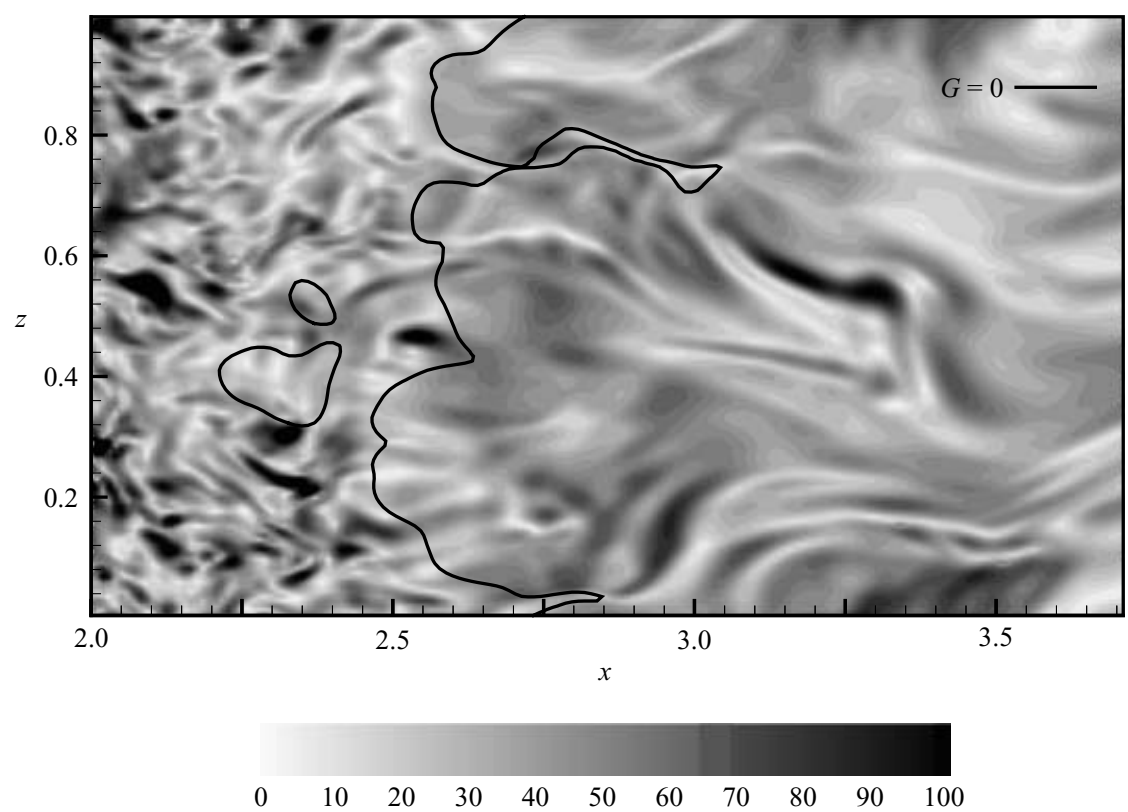

FIGURE 23. The modulus of the vorticity, $\sqrt{\omega_{i}^{2}}$, plotted in an instantaneous slice in the $(x, z)$-plane. $\tau=3$.

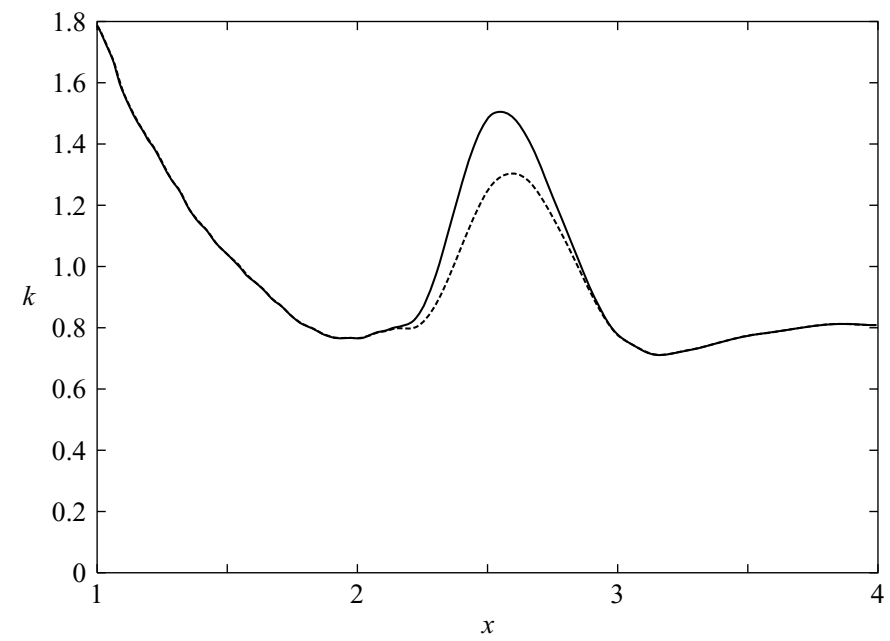

FIgURE 24. ---, The Favre-averaged turbulent kinetic energy, compared to - , the normal averaged turbulent kinetic energy. $\tau=3$.

\section{Conclusions}

We have carried out a direct numerical simulation of a premixed flame in decaying homogeneous turbulence. The simulation includes the effects of gas expansion in a flow at low Mach number. The flame front is modelled by a level set approach. In this way, it is possible to perform an efficient simulation, which includes the most important phenomena of premixed turbulent combustion. It is possible to stabilize the mean 
position of the flame front by adjusting the inflow velocity into our computational domain. This allow us to obtain converged statistics by means of time averaging.

The DNS is able to reproduce hydrodynamic instability of the flame front. The dependency of the growth rate on the expansion rate and on the wavelength of the initial wavelike perturbation is in good agreement with the theory. When the thickness of the flame front increases, the growth rates become lower than the theory because the theory is only valid for infinitely thin flames.

The turbulent flame speed increases with increasing turbulence intensities at the position of the flame front. This is due to the deformation of the flame front by the turbulence with a result that its area increases, leading to a larger combustion rate. The rise in flame speed agrees with theoretical and experimental results. Apart from the direct effect of turbulent deformation of the flame front, the increase is found to depend also on the heat release. For higher expansion rates, the increase of the flame speed grows. This effect is due to the hydrodynamic instability mechanism, which results in an amplification of the disturbances that are induced on the flame front by the turbulence.

When passing the flame front, the turbulent kinetic is distributed over a larger volume as a result of the gas expansion, which accompanies the heat release. This means that turbulent kinetic energy decreases. At the higher expansion rates, however, we find that turbulent kinetic energy can also be produced. The production process is related to flame-front deformation, which as a result of the hydrodynamic instability amplifies and produces large velocity fluctuations in the streamwise direction. As a result, the total turbulent kinetic energy increases (despite the decay due to the gas expansion). Because the production affects mainly one flow direction, the turbulence exhibits an anisotropic structure after the flame front. We note, however, that the imposed turbulence at the flame front in our case is rather small, which perhaps allows the instability process to dominate. This might be different at higher turbulence levels as argued by Boughanem \& Trouvé (1998).

Vorticy at the flame front decreases as a result of the same process that we have observed for the kinetic energy, i.e. the distribution over a larger volume owing to the gas expansion. Vorticity, however, is also generated at the flame front by baroclinic production, which is due to the non-alignment of pressure and density gradients. For high expansion rates, this production will become stronger than the suppression by the gas expansion and this causes the vorticity to increase over the flame front for larger expansion rates. Furthermore, we find that the vorticity after the flame front has an elongated anisotropic structure.

Turbulence in compressible flows at low Mach number or alternatively nonconstant-density flows is frequently treated in terms of so-called Favre averaging. Because density fluctuations play an important role in the production of turbulent kinetic energy at the position of the flame front, standard Favre averaging where additional terms in the kinetic energy budget are neglected, fails. To model properly the dynamics of the flame front and the interaction between the flame front and the turbulent flow, these additional terms in the Favre-averaged kinetic energy budget must be modelled.

The authors wish to thank N. Peters, R. Klein and H. Wenzel for the useful discussion during our visit to the university of Aachen and the National Computing Facilities Foundation (NCF) for providing the computing resources needed for this work. The comments on the manuscript by Professor J.C.R. Hunt and by the anonymous reviewers have been very helpful. 


\section{REFERENCES}

Ashurst, W. 1997 Darrieus-Landau instability, growing cycloids and expanding flame acceleration. Combust. Theory Modelling 1, 405-428.

Batchelor, G. K. 1967 An Introduction to Fluid Dynamics. Cambridge University Press.

Boughanem, H. \& TrouvÉ, A. 1998 The occurence of ame instabilities in turbulent premixed combustion. In Twenty-Seventh Symposium (International) on Combustion. The Combustion Institute.

Bray, K., Champion, M. \& Libby, P. 2000 Premixed flames in stagnating turbulence, part iv, a new theory for the Reynolds stresses and Reynolds fluxes applied to impinging flows. Combust. Flame 120, 1-18.

Brethouwer, G. 2001 Mixing of passive and reactive scalars in turbulent flows. PhD thesis, Delft University of Technology.

DamköHLER, G. 1940 Der Einfluß der Turbulenz auf die Flammengeschwindigkeit in Gasgemischen. Z. Elektrochem. 46, 601-652.

George, W. 1992 The decay of homogenous turbulence. Phys. Fluids 4, 1492-1509.

Ibrahim, S. S., Hargrave, G. K. \& Williams, T. C. 2001 Experimental investigation of flame/solid interactions in turbulent premixed combustion. Exp. Thermal Fluid Sci. 24, 99-106.

Im, H., Lund, T. \& Ferziger, J. 1995 Study of turbulent premixed flame propagation using a laminar flamelet model. In Annu. Res. Briefs 1995, pp. 347-360. Center for Turbulence Research, Stanford.

Im, H. G., Lund, T. S. \& Ferziger, J. H. 1997 Large eddy simulation of turbulent front propagation with dynamic subgrid models. Phys. Fluids 9, 3826-3833.

Landau, L. 1944 On the theory of slow combustion. Acta Physicochim. pp. 19-77.

Landau, L. \& Lifshitz, E. M. 2000 Fluid Mechanics, 2nd edn. Butterworth-Heinemann.

Lele, S. K. 1994 Compressibility effects on turbulence. Annu. Rev. Fluid Mech. 26, 211-254.

LindSTEDT, R. \& SAKTHITHARAN, V. 1998 Time resolved velocity and turbulence measurements in turbulent gaseous explosions. Combust. Flame 114, 469-483.

LOUCH, D. \& BRAY, K. 1998 Vorticity and scalar transport in premixed turbulent combustion. In Twenty-Seventh Symposium (International) on Combustion, pp. 801-810. The Combustion Institute.

Markstein, G. 1964 Nonsteady Flame Propagation. Macmillan.

Moin, P. \& Mahesh, K. 1998 Direct numerical simulation: a tool in turbulence research. Аnnu. Rev. Fluid Mech. 30, 539-578.

Mueller, C. J., Driscoll, J. F., Reuss, D. L., Drake, M. C. \& Rolik, M. E. 1998 Voriticity generation and attenuation as vortices convect through a premixed flame. Combust. Flame 112, 342-358.

NaJM, H. N., Wyckoff, P. S. \& KNiO, O. M. 1998 A semi-implicit numerical scheme for reacting flow, i. stiff chemistry. J. Comput. Phys. 143, 381-402.

Peters, N. 2000 Turbulent Combustion. Cambridge University Press.

Peters, N., Wenzel, H. \& Williams, F. 2000 Modification of the turbulent burning velocity by gas expansion effects. In Twenty-Eighth Symposium (International) on Combustion. The Combustion Institue.

Piana, J., Veynante, D. \& Candel, S. 1996 Direct numerical simulation analysis of the $g$ equation in premixed combustion. In Direct and Large Eddy Simulation II, pp. 321ff. Kluwer, Dordrecht.

Plessing, T., Kortschik, C., Mansour, M. \& Peters, N. 2000 Measurements of the turbulent burning velocity and the flame structure of premixed flames on a low swirl burner. In Twenty-Eighth Symposium (International) on Combustion. The Combustion Institue.

Poinsot, T. \& Veynante, D. 2001 Theoretical and Numerical Combustion. Edwards.

Poinsot, T., Veynante, D. \& CANDEL, S. 1991 Quenching processes and premixed turbulent combustion diagrams. J. Fluid Mech. 228, 561-606.

Poinsot, T., CANDEl, S. \& Trouvé, A. 1996 Applications of direct numerical simulation to premixed turbulent combustion. Prog. Energy Combust. Sci. 21, 531-576.

Rutland, C. \& Cant, S. 1994 Turbulent transport in premixed flames. In Proc. Summer Program, 75-94, Center for Turbulence Research. 
Rutland, C. \& Ferziger, J. 1991 Simulations of flame-vortex interactions. Combust. Flame 84, 343-360.

Sethian, J. 1999 Level Set Methods and Fast Marching Methods. Cambridge University Press.

Sussman, M., Smereka, P. \& Osher, S. 1994 A level set approach for computing solutions to incompressible two-phase flow. J. Comput. Phys. 114, 146-159.

Wenzel, H. \& Peters, N. 1998 Direct numerical simulation of premixed turbulent combustion using a flamelet approach. In Notes on Numerical Fluid Mechanics: Computation and visualization of three-dimensional vortical and turbulent flows (ed. R. Friedrich \& P. Bontoux), 64, 276-296.

Wenzel, H. \& Peters, N. 2000 Direct numerical simulation and modeling of kinematic restoration, dissipation and gas expansion effects of premixed flames in homogeneous turbulence. Combust. Sci. Techno.

Williams, F. 1985a Turbulent combustion. In The Mathematics of Combustion (ed. J. Buckmaster), pp. 97-131. SIAM, Phildelphia.

Williams, F. A. 1985b Combustion Theory. Perseus Books, Reading.

Wirth, M., Keller, P. \& Peters, N. 1993 A flamelet model for permixed turbulent combustion in si-engines. SAE Paper 932646.

Zeldovich, Y., Barenblatt, G., Librovich, V. \& Makhivladze, G. 1985 The Mathematical Theory of Combustion and Explosions. New York.

Zhang, S. \& RutLand, C. J. 1995 Premixed flame effects on turbulence and pressure-related terms. Combust. Flame 102, 447-461. 